\title{
1 Resistome diversity in bovine clinical mastitis microbiome, a signature
}

3 M. Nazmul Hoque ${ }^{1,2}$, Arif Istiaq ${ }^{1,3}$, Rebecca A. Clement ${ }^{4}$, Keylie M. Gibson ${ }^{4}$, Otun Saha ${ }^{1}$,

4 Ovinu Kibria Islam ${ }^{1,5}$, Ruhshan Ahmed Abir ${ }^{6}$, Munawar Sultana ${ }^{1}$, AMAM Zonaed Siddiki ${ }^{7}$,

5 Keith A. Crandall ${ }^{8}$, M. Anwar Hossain ${ }^{1,9, *}$

$6{ }^{1}$ The Laboratory of Microbial Genetics and Bioinformatics, Department of Microbiology,

7 University of Dhaka, Dhaka 1000, Bangladesh

$8 \quad{ }^{2}$ Department of Gynecology, Obstetrics and Reproductive Health, Faculty of Veterinary

9 Medicine and Animal Science, Bangabandhu Sheikh Mujibur Rahman Agricultural

10 University, Gazipur-1706, Bangladesh

$11{ }^{3}$ Department of Developmental Neurobiology, Graduate School of Medical Sciences,

12 Kumamoto University, Kumamoto, Japan

$13{ }^{4}$ Computational Biology Institute, Milken Institute School of Public Health, The George

14 Washington University, USA

$15{ }^{5}$ Department of Microbiology, Jashore University of Science and Technology, Jashore 7408,

16 Bangladesh

$17{ }^{6}$ Bio-Bio-1, Bioinformatics Research Foundation, Bangladesh

$18{ }^{7}$ Department of Pathology and Parasitology, Chittagong Veterinary and Animal Sciences

19 University, Chittagong-4202, Bangladesh

$20{ }^{8}$ Department of Biostatistics and Bioinformatics, Milken Institute School of Public Health,

21 The George Washington University, USA

$22{ }^{9}$ Present address: Vice-Chancellor, Jashore University of Science and Technology, Jashore

237408 , Bangladesh

$24 *$ *Correspondence to: hossaina@du.ac.edu 


\section{Abstract}

27 The bovine clinical mastitis (CM) milk is a large reservoir for diverse groups of resistomes, 28 which play important roles in the pathogenesis of mastitis, but little is known about the 29 concurrence of CM microbiome signature and its associated resistomes. Here we deciphered

30 the total resistance (antibiotics and metals resistance, biofilm formation, quorum sensing)

31 present in CM microbiome using whole metagenome sequencing (WMS) and in vitro cultural 32 approaches. Significant correlation $(p=0.001)$ was found between the resistome diversity and 33 microbiome signature. We identified the strain-level microbiome diversity in four cattle 34 breeds, with microbiome composition represented by the phyla Proteobacteria, 35 Bacteroidetes, Firmicutes, Actinobacteria and Fusobacteria (contributing to $>95.0 \%$ of total 36 strains). However, the resistome diversity did not vary significantly ( $p=0.692)$ across the 37 microbiomes of cattle breeds. The in vitro investigation showed that biofilm producing CM 38 pathogens were resistant to most of the conventional antibiotics used for CM treatment, 39 whereas these pathogens remained sensitive to five heavy metals $(\mathrm{Cr}, \mathrm{Co}, \mathrm{Ni}, \mathrm{Cu}, \mathrm{Zn})$ at 40 varying concentrations. We also found association of some genomic functional potentials 41 such as bacterial flagellar movement and chemotaxis, regulation and cell signaling, phages42 prophages, transposable elements, plasmids and oxidative stress in the pathophysiology of 43 bovine $\mathrm{CM}$. These findings of rapid and reliable identification of $\mathrm{CM}$ microbiomes and 44 associated resistomes will help improve the optimization of therapeutic schemes involving 45 antibiotics and metals usage in the prevention and control programs of bovine CM.

49 Key words: Clinical mastitis, diversity, resistome, microbiome. 


\section{Introduction}

Mastitis is the foremost production and major economic burden confronted by the global dairy industry ${ }^{1-3}$. Bovine clinical mastitis $(\mathrm{CM})$ is of special concern for milk producers in developing countries like Bangladesh, where dairying plays a pivotal role in the national economy. The CM milk from dairy animals is now considered to host a complex microbial community with great diversity ${ }^{2-4}$. The most frequently isolated pathogens are Staphylococcus aureus, Escherichia coli, Klebsiella spp., Streptococcus spp., Mycoplasma spp., Enterobacter spp., Bacillus spp., Corynebacterium species $^{5-8}$. Therefore, accurate identification of pathogens causing $\mathrm{CM}$ enables appropriate choices for antimicrobial treatment and preventive mastitis management ${ }^{8-10}$. Over the past two decades, a wide range of phenotyping and genotyping methods have been implemented to study mastitis-causing bacteria $^{6-9}$. Although culture-based techniques are in the forefront of detecting CM bacteria, these methods are time-consuming and have inherent drawback of not being applicable to non-cultivable bacteria ${ }^{11}$. Until recently, $16 \mathrm{~S}$ rRNA partial gene sequencing remained as the most commonly used genomic survey tool to study bovine mastitis microbiomes ${ }^{3,4,12}$. However, this technique has limitations because of polymerase chain reaction (PCR) bias, lower taxonomic resolution at the species level, and limiting information on gene abundance and functional profiling ${ }^{13}$. Shotgun whole metagenome sequencing (WMS), on the other hand, produces a metagenome reflecting the breadth of microbial genomic content in a sample and successfully provides insights into the phylogenetic composition, species and/or strain and functional diversity for a variety of biomes ${ }^{2,13,14}$. This WMS typically produces high complexity datasets with millions of short reads allowing extensive characterization of microbiome in an ecological niche ${ }^{13,14}$ and profiling of their functional attributes like microbial energy metabolism, antimicrobial resistance and biofilm forming abilities; and gradually becoming a cost-effective metagenomic approach ${ }^{13}$. The cattle breeds or host 
76 genetics may have an influence on the milk microbiota composition and on susceptibility to

77 disease and resistance to bacterial infection ${ }^{12,15}$. The milk from healthy Holstein Friesian

78 cows displayed more significant changes bacterial biodiversity and composition than

79 microbiota in Rendena cows milk ${ }^{12,16}$.

80 The secretion of antimicrobial compounds by microbes is an ancient and effective

81 method to improve the survival of microbes competing for space and nutrients with other

82 microorganisms ${ }^{17}$. However, the advent recent metagenomic studies have revealed

83 diverse homologues of known resistance genes broadly distributed across

84 environmental locales including bovine milk samples. This widespread dissemination

85 of antimicrobial resistance elements is inconsistent with a hypothesis of contemporary

86 emergence and instead suggests a richer natural history of resistance ${ }^{18}$. The vast

87 diversity of bacterial species in CM milk coupled with short generation times and horizontal

88 gene transfer permit the rapid accumulation of countless resistance variations at a relatively

89 high evolutionary pace ${ }^{19}$. Resistance in CM bacteria typically goes unnoticed until a given

90 species becomes of clinical interest, and the resistome found CM is also suspected to be a

91 source of newly emerging resistance genes in the $\mathrm{CM}^{2,8,17,20}$. Antibiotics have been used for

92 decades in livestock production for both therapeutic (e.g. treatment of specific diseases) and

93 nontherapeutic (growth promotion) $\operatorname{purposes}^{10}$. However, there are data that support the fact

94 that both nontherapeutic and therapeutic doses of antibiotics can contribute to the emergence

95 of antimicrobial-resistant bacteria, thus exacerbating the problem of antibiotic resistance in

96 animal and human pathogens ${ }^{10}$, and enhancing the selection for antibiotic resistance genes

97 (ARGs) and the horizontal transfer of these genes ${ }^{10,17}$. Bacteria residing in the bovine

98 gastrointestinal tract and udder may become resistant to these antibiotics and, once released

99 into the milk, they may transfer ARGs to other CM bacteria of contagious and environmental

100 origin $^{8,20}$. Efficacy of antimicrobial therapy against bovine CM pathogens is low ${ }^{8}$, and the use 
101 of antibiotics, confined to selected severe CM cases necessitates the accurate identification

102 and characterization of pathogens and antibiotic selection for its better prevention and 103 control $^{1,8}$. Furthermore, antimicrobial resistance (AMR) is a global health concern in both

104 human and veterinary medicine ${ }^{10}$, and thus, monitoring the emergence of AMR bacterial

105 strains is an essential component of bovine CM prevention and control strategies ${ }^{8,21}$.

106 Therefore, finding an effective alternative strategy for the control of bovine mastitis is a 107 challenge for dairy producers.

108 The antimicrobial properties of metals have been documented throughout the history 109 of medicine and healthcare ${ }^{22}$. The metal salts such as chromium (Cr), cobalt (Co), nickel (Ni),

110 copper $(\mathrm{Cu})$ and zinc $(\mathrm{Zn})$ are effective in controlling bacterial transmission and infection

111 risks $^{22}$. However, their uses are limited due to their toxicity and possible detrimental

112 environmental effects in dairy industries particularly as therapeutic agents against bovine CM

113 pathogens. Biofilm formation is an important virulence factor for mastitis causing bacteria

114 and contributes to the resistance to different classes of antimicrobials ${ }^{23}$. Bacterial pathogens

115 identified in this study showed broad spectrum of antimicrobial (antibiotics, toxic metals)

116 resistance, and possessed biofilm forming and quorum sensing abilities, which might be the

117 potential factors hindering CM cures, thereby leading to the persistence of the disease, and 118 increased risk of transmission to non-infected dairy cows. Genetic information about 119 resistance or in vitro assays of resistance is not enough to understand about resistomes when 120 considered solely rather in combination ${ }^{10,11}$. Genetic potential doesn't give the idea of 121 resistance level as many other factors are involve such as expression, stimulation, stress 122 etc $^{10,11,15}$. Similarly, resistance assay doesn't give the idea about genetic makeup responsible.

123 Therefore, our present study describes the resistome diversity across microbial communities 124 causing CM in four major cattle breeds (Local Zebu, LZ; Red Chattogram Cattle, RCC;

125 Sahiwal, SW; Crossbred Holstein Friesian; XHF) of Bangladesh using both metagenomic 
126 deep sequencing (WMS) and in vitro cultural approaches. Furthermore, we also aimed to

127 investigate the influences of metabolic genomic potentials of the microbiomes in the 128 pathophysiology of bovine CM.

\section{$130 \quad$ Results}

131 To decipher the resistome diversity in bovine CM microbiomes, we used a condition of 132 combination of in silico (WMS, 16S rRNA gene sequencing) and in vitro (culture base) 133 approaches. The present WMS investigation leads to the direct and comprehensive evaluation

134 of resistance to antibiotics and toxic compounds (RATC), biofilm formation (BF) and 135 quorum sensing (QS) genes in $25 \mathrm{CM}$ samples. Furthermore, in vitro antimicrobial resistance 136 profiling of six CM causing bacteria (S. aureus, E. coli, Klebsiella, Enterobacter, Bacillus 137 and Shigella) isolated from 260 milk samples was carried out using 12 commonly used 138 antibiotics (ampicillin, doxycycline, tetracycline, nitrofurantoin, ciprofloxacin, nalidixic acid, 139 cefoxitin, imipenem, chloramphenicol, gentamycin, erythromycin, vancomycin), and five 140 toxic metals (copper, zinc, chromium, nickel, cobalt). Moreover, we also demonstrated some 141 functional metabolic potentials of CM microbiomes found to be associated with mammary 142 gland pathogenesis.

\section{Sequence analysis}

144 The WMS of $25 \mathrm{CM}$ milk samples generated approximately 600 million reads, ranging 145 from 8.86 to 39.75 million per sample. An average of 21.13 million reads per sample 146 (maximum=36.89 million, minimum=4.71 million) passed the quality control step

147 (Supplementary Data 1). We analyzed the sequencing reads simultaneously using two 148 bioinformatics pipelines, PathoScope 2.0 (PS) and MG-RAST (MR). 


\section{Microbiome diversity and composition in CM}

152 We investigated the strain-level microbial community and relative abundances in $25 \mathrm{CM}$ milk 153 samples (previously published 14 samples $^{2}$ and 11 new samples) through WMS. The reads 154 generated from WMS mapped to 391 genera and 519 strains of bacteria through MR and PS 155 analyses, respectively (Supplementary Data 1).

156 The rarefaction curves based on observed species richness reached a plateau after, on 157 average, 23.87 million reads (Fig. 1a, Supplementary Data 1)-suggesting that the depth of coverage for most samples was sufficient to capture the entire microbial diversity within each sample. Although, we did not find any significant differences in the alpha (observed species,

160 Chao1, ACE, Shannon, Simpson and Fisher diversity estimates) and beta (based on Bray-

161 Curtis dissimilarity matrix) diversities among the microbial communities across the $25 \mathrm{CM}$ 162 samples (Fig. 1b,c). However, significant diversity (alpha and beta) differences were 163 observed among the CM microbiome communities across the four cattle breeds (LZ, RCC, 164 SW, XHF) regardless of the method (i.e., either PS or MR) used to tabulate microbial abundances (PS; $p=0.005, \mathrm{MR} ; p=0.001$, Kruskal-Wallis test). In addition, this breed specific diversity difference remained evident in the microbial ecosystem of XHF cows associated

167 CM milk samples (Fig. 1d,e). The PCoA analysis also showed significant microbial disparity 168 ( $p=0.001)$ among the microbiome of four dairy breeds (Fig. 1e).

169 The predominant bacterial phyla were Proteobacteria, Bacteroidetes, Firmicutes, 170 Actinobacteria and Fusobacteria (contributing to $>95.0 \%$ of the total sequences, Kruskal171 Wallis test, $p=0.001)$ in the MR analysis. The strain-level signature of the microbiome 172 demonstrated that most of the species identified in each CM sample represented by multiple

173 strains (Supplementary Data 1), and of the detected bacterial strains, the top 200 strains 174 (according to their relative abundance) are depicted in Fig. 2. The CM associated microbiome 175 was dominated by 29 different strains of Pseudomonas species, while Acinetobacter, 
176 Streptococcus, Lactobacillus, Corynebacterium, Staphylococcus and Enterococcus species

177 represented by 27, 27, 18, 17, 15 and 10 different strains, respectively (Fig. 2, Supplementary

178 Data 1). Thus, among the identified bacterial strains, A. johnsonii XBB1 had the highest 179 relative abundance (38.9\%) and followed by Micromonospora sp. HK10 (17.6\%). Other 180 bacterial strains found abundantly were Campylobacter mucosalis (8.7\%), P. putida KT2440

181 (7.7\%), Anaerobutyricum hallii DSM 3353 (6.3\%), P. fragi (3.2\%), Catenibacterium 182 mitsuokai DSM 15897 (3.0\%), E. coli O104:H4 str. 2011C-3493 (2.0\%), A. veronii (1.2\%), 183 Pantoea dispersa EGD-AAK13 (1.1\%), P. fluorescens Pf0-1 (0.8\%), K. oxytoca (0.7\%) and 184 P. entomophila L48 (0.5\%). The remaining strains had a relatively lower abundance $(<0.5 \%)$ 185 (Supplementary Data 1). According to the cattle breeds, the XHF cows had the highest 186 number of microbial strains $(n=403)$ followed by LZ cows $(n=230)$, SW cows $(n=134)$ and 187 RCC (n=125) (Fig. 3a-c, Supplementary Data 1). The breed specific association revealed that 188 45.7, 22.6 and $19.1 \%$ of the detected bacterial strains in CM milk samples of LZ, SW and 189 RCC cows, respectively, were also found in the CM microbiome of XHF cows (Fig. 3d, 190 Supplementary Data 1).

191 Simultaneously through in vitro cultural analysis, a total of 452 isolates that belonged 192 to six bacterial (S. aureus, E. coli, Klebsiella, Enterobacter, Bacillus and Shigella) species 193 were identified in 260 CM samples (including 25 WMS CM samples) collected from central $194(\mathrm{CR}=160)$ and southeastern (SER=100) regions of Bangladesh (Supplementary Fig. 1). The 195 overall prevalence of S. aureus, E. coli, Klebsiella, Enterobacter, Bacillus and Shigella 196 species were 23.5, 18.5, 19.2, 12.3, 9.2 and 17.3\% CM samples, respectively (Supplementary 197 Table 1). We found significant differences in the prevalence of these species $(p=0.01)$ when 198 analyzing the distribution of these pathogens according to the origin of the samples (SER and 199 CR) (Supplementary Fig. 2). The culture-based findings of the current study demonstrated $S$. 200 aureus as the chief etiology of bovine CM in Bangladesh, while Shigella species remained as 
201 the least frequently detected CM pathogen - which corroborates with the results of WMS-

202 based taxonomic identification (Supplementary Fig. 3).

\section{Resistomes diversity and composition of CM microbiome}

204 For analyses of resistome diversity and abundance in CM microbiomes, the SEED 205 module of the MR pipeline provided a comprehensive picture. Using SEED, 147,040 reads 206 aligned to 30 resistance to antibiotics and toxic compounds (RATC) and 10 biofilm

207 formation and quorum sensing (BF-QS) functional groups across the CM samples with 208 different abundances (Supplementary Data 2). The RATC genes classified into two unique 209 groups, 19 antibiotic resistance and 11 toxic metal resistance groups (Fig. 4, Supplementary

210 Data 2). This WMS analysis showed significant correlation (Pearson correlation, $p=0.001$;

211 Nonparametric Spearman's Correlation, $p=0.003$ ) between the number of reads aligned to 212 bacterial genomes and number of reads mapped to RATC genes (Supplementary Data 2).

213 Among the RATC functional groups, multidrug resistance to efflux pumps (MREP, 28.6\%),

214 CmeABC operon (8.9\%), resistance to fluoroquinolones (RFL, 6.2\%), mdtABCD cluster 215 (5.5\%), methicillin resistance in Staphylococci (MRS, 3.8\%), BlaR1 regulatory family

$216($ BlaR1, 3.4\%), MexE-MexF-OprN (2.4\%) and beta-lactamase resistance (BLAC, 2.2\%) were

217 the dominating antibiotic resistance genes (ARGs) found in CM milk microbiomes (Fig. 4a,

218 Supplementary Data 2). In addition to ARGs, the WMS analysis also detected a number of

219 metal and toxic compound resistance (MTR) genes in CM microbiomes. Among them, 220 cobalt-zinc-cadmium resistance (CZCR, 19.3\%), copper homeostasis $(\mathrm{CH}, 9.6 \%)$, arsenic 221 resistance (AR, 2.9\%), copper homeostasis: copper tolerance (CHCT, 2.3\%) and resistance to 222 chromium compounds (RCHC, 1.4\%) were the predominating resistant genes (Fig. 4a, 223 Supplementary Data 2). Although the relative abundance of these RATC genes varied 224 among the microbiomes of the four breeds (LZ, RCC, SW and XHF), but their resistome 
225 diversity did not vary significantly $(p=0.692)$ by taxonomic diversity of respective breeds

226 (Fig. 4b, Supplementary Data 2).

The diversity and composition of RATC functional groups also varied significantly $(p=0.027)$ in in vitro selected six CM pathogens isolated and identified from different sources of CM samples (breed and study areas) under almost same farming management system (Fig.

230 5a, Supplementary Data 2). Among the RATC groups, the predominant ARGs found as 231 follows MRS (S. aureus, 37.0\%), RFL (S. aureus, 14.8\%; Shigella, 7.8\%), MREP (E. coli, 232 28.5\%; Klebsiella, 28.4\%), BlaR1 (E. coli, 6.0\%; Shigella, 8.5\%), mdtABCD cluster (E. coli, $23317.5 \%$; Klebsiella,18.9\%; Enterobacter, 21.4\%; Shigella, 11.7\%), multiple antibiotic 234 resistance (MAR) Locus (E. coli, 2.4\%; Enterobacter, 2.6\%), CmeABC operon (E. coli, $2359.1 \%$; Enterobacter, 11.0\%; Shigella, 25.6\%), and adaptation to d-cysteine, ADCYS 236 (Bacillus, 5.5\%) (Fig. 5b). Conversely, genes encoding $\mathrm{CH}$ in S. aureus (11.1\%), E. coli 237 (4.8\%), Enterobacter (4.4\%), and Shigella (6.0\%), CHCT in Klebsiella (11.2\%) and Shigella 238 (3.7\%), mercuric reductase (MRD) in S. aureus (11.1\%), mercury resistance to operon 239 (MROP) in Enterobacter (2.4\%), AR in S. aureus (3.7\%), E. coli (4.4\%), Klebsiella (10.1\%), 240 Enterobacter (7.5\%) and Shigella (7.8\%), ZR in E. coli (5.6\%), cadmium resistance (CDR) in 241 S. aureus (3.7\%), CZCR in S. aureus (3.7\%), E. coli (10.4\%), Klebsiella (11.6\%), 242 Enterobacter (20.3\%) and Shigella (21.0\%), and RCHC in Bacillus (85.0\%) were the most 243 abundant toxic compounds or metals resistant (MTR) RATC functional groups among the six 244 selected pathogens (Fig. 5c). Assessment of the BF-QS ability of the CM microbiomes 245 revealed that autoinducer 2 (AI-2) transport and processing (lsrACDBFGE operon, 33.7\%), 246 biofilm adhesion biosynthesis (BAB, 24.2\%), protein YjgK cluster linked to biofilm 247 formation ( $\mathrm{jgK}$ cluster, 15.5\%), quorum sensing: autoinducer-2 synthesis (QSAU2, 9.4\%) 248 were the most abundant genes among CM associated pathogens (Supplementary Data 2). 249 However, by comparing the association of these BF-QS genes among the selected six 
250 bacterial pathogens, we found significant variation $(p=0.017)$ in their diversity, composition 251 and relative abundances (Fig. 5d, Supplementary Data 2).

252 The in vitro antibiogram profiling of 221 individual isolates of the six bacteria 253 revealed that $S$. aureus isolates had highest resistance to doxycycline, ampicillin, tetracycline 254 and erythromycin (73.0 to $88.0 \%$ ) and moderate resistance to chloramphenicol, ciprofloxacin 255 and nitrofurantoin (50.0 to 58.0\%) (Fig. 6, Table 1). The isolates of another Gram-positive 256 bacterium (Bacillus) demonstrated highest resistance against doxycycline, ampicillin, 257 nalidixic acid and erythromycin (60.0 to 84.0\%). However, E. coli isolates exhibited highest 258 resistance against tetracycline, doxycycline, nalidixic acid and ampicillin (77.0 to 93.0\%) and 259 moderate resistance to chloramphenicol, nitrofurantoin, gentamicin and ciprofloxacin (40.0 to $26063.0 \%$ ). The isolates of Klebsiella, Enterobacter and Shigella species displayed highest 261 resistance to doxycycline, nalidixic acid, tetracycline and ampicillin (70.0 to 100.0\%) and 262 moderate resistance to ciprofloxacin, gentamicin, nitrofurantoin and chloramphenicol (30.0 to $26370.0 \%$ ). In this study, imipenem and cefoxitin remained as the most sensitive antibiotics 264 against four Gram-negative bacterial (E. coli, Klebsiella, Enterobacter and Shigella) species, 265 while the two Gram-positive ( $S$. aureus and Bacillus) species were mostly sensitive to 266 imipenem, cefoxitin and vancomycin (Fig. 6, Table 1). Taken together, the antibiogram 267 profile revealed that all of the selected CM pathogens are becoming multidrug resistant 268 (MDR, resistant to $\geq 5$ antibiotics) and the highest resistance was found to tetracyclines 269 (tetracycline and doxycycline) followed by quinolones (nalidixic acid) and penicillin 270 (ampicillin) groups of antibiotics (Fig. 6, Table 1).

271 The use of heavy metals in soluble forms as an alternative to prevent bovine CM 272 appears as a novel promising idea supported by several earlier studies ${ }^{1,22}$. Zones of inhibition 273 (ZOI) assays using the individual metal solution $(\mathrm{Cu}, \mathrm{Zn}, \mathrm{Cr}$, Co and $\mathrm{Ni})$ demonstrated an 274 increase in antimicrobial activity which correlated with increased metal ion solution 
275 concentration $(p<0.001)$ (Fig. 7). Thus, ZOI assays of metals demonstrated S. aureus (ZOI:

$27625.4 \mathrm{~mm}$ ) as the most sensitive CM pathogens followed by Bacillus (ZOI: $23.4 \mathrm{~mm}$ ), E coli

277 (ZOI: $20.6 \mathrm{~mm})$, Enterobacter (ZOI:18.9 mm), Klebsiella (ZOI:17.8 mm) and Shigella

278 (ZOI:15.4 mm) species (Fig. 7a). The minimal inhibitory concentration (MIC) of the metal

279 ions demonstrated a varying degree of response against all the tested CM pathogens, and

280 these bacteria tolerated a wide range of metal concentration (3.4 to $38.1 \mu \mathrm{g} / \mathrm{mL}$ )

281 (Supplementary Data 2). We compared the highest MIC values of each metal, and found that

282 highest MIC values decrease in the following order: $\mathrm{Zn}(38.1 \mu \mathrm{g} / \mathrm{mL}$, S. aureus), $\mathrm{Cu}$ (33.2

$283 \mu \mathrm{g} / \mathrm{mL}$, S. aureus $), \mathrm{Ni}(28.2 \mu \mathrm{g} / \mathrm{mL}$, E. coli $), \mathrm{Cr}(17.2 \mu \mathrm{g} / \mathrm{mL}$, Enterobacter species), and Co

284 (15.3 $\mu \mathrm{g} / \mathrm{mL}$, Bacillus spp.) (Fig. 7b, Supplementary Data 2). For the MIC of specific

285 bacteria, the most effective metals were found to be $\mathrm{Cr}$ against Shigella $(3.4 \mu \mathrm{g} / \mathrm{mL})$ and

286 Klebsiella $(5.8 \mu \mathrm{g} / \mathrm{mL})$ species, Ni against Shigella $(3.5 \mu \mathrm{g} / \mathrm{mL})$ species, Co against Shigella

$287(5 \mu \mathrm{g} / \mathrm{mL})$ and Klebsiella $(7.4 \mu \mathrm{g} / \mathrm{mL})$ species, and $\mathrm{Cu}$ and $\mathrm{Zn}$ against Shigella $(7.5 \mu \mathrm{g} / \mathrm{mL}$,

288 both) species. In contrast, $\mathrm{Zn}(38.1 \mu \mathrm{g} / \mathrm{mL})$ and $\mathrm{Cu}(33.2 \mu \mathrm{g} / \mathrm{mL})$ were the least toxic metals

289 against S. aureus (Fig. 7b, Supplementary Data 2). A similar pattern was demonstrated for the

290 minimal bactericidal concentration (MBC) with the greatest bactericidal activity for $\mathrm{Cr}$

291 against $S$. aureus $(11.3 \mu \mathrm{g} / \mathrm{mL})$ followed by Co against $E$. coli $(14.3 \mu \mathrm{g} / \mathrm{mL})$, Ni against $S$.

292 aureus $(23.1 \mu \mathrm{g} / \mathrm{mL}), \mathrm{Zn}$ against E. coli $(24.2 \mu \mathrm{g} / \mathrm{mL})$, and $\mathrm{Cu}$ against Shigella $(25.1 \mu \mathrm{g} / \mathrm{mL})$

293 species. However, $\mathrm{Cu}$ produced equable antimicrobial efficacy as $\mathrm{Zn}, \mathrm{Cr}$, Co and $\mathrm{Ni}$ against

294 Enterobacter species $(\leq 25.5 \mu \mathrm{g} / \mathrm{mL})$ (Supplementary Table 2$)$.

295 To assess BF ability of CM pathogens in in vitro condition, we randomly selected 80

296 isolates (S. aureus, 15; E. coli, 15; Klebsiella, 15; Bacillus, 15; Enterobacter, 10 and Shigella,

297 10) for BF assay. In this study, 76.2\% (61/80) bacterial species were biofilm producers with

298 significance differences $(p=0.028)$, and their categories of BF were strong biofilm forming

299 (SBF, 28.7\%), moderate biofilm forming (MBF, 25.2\%), weak biofilm forming (WBF, 
$30022.2 \%$ ) and non-biofilm forming (NBF, 23.7\%) (Fig. 8). While investigated individually, $E$.

301 coli $(66.7 \%)$ remained as the highest biofilm producing CM pathogen followed by

302 Enterobacter (60.0\%), Klebsiella (46.7\%), S. aureus (40.0\%), Shigella (30.0\%) and Bacillus

303 (26.7\%) species. Our current findings revealed that Gram-negative CM pathogens

304 (Enterobacter, 60.0\%; E. coli, 40.0\%; Shigella, 33.3\%; Klebsiella, 28.6\%) had higher biofilm

305 producing ability than Gram-positive bacteria (S. aureus, 16.7\%) (Fig. 8a,b). On the contrary,

306 the majority of the Bacillus (73.3\%), Shigella (70.0\%) and S. aureus (60.0\%) isolates

307 remained as non-biofilm formers (NBF) (Fig. 8b). Therefore, our current findings of in vitro

308 resistance analysis (antibiotics and metals resistance and biofilm assays) corroborate the

309 resistome found in metagenome sequencing.

\section{Pathogenic functional potentials genome of the CM microbiomes}

311 We also investigated the possible links between chemotaxis and pathogenicity

312 through the identification of putative genes or proteins associated with both flagellar motility

313 and bacterial chemotaxis. The KEGG pathway analysis of MR tool identified 48 protein

314 families associated with flagellar motility in prokaryotes, and among them, flagellar hook-

315 length control protein, FliK (27.1\%); flagellar biosynthesis proteins, FlhA, FliL, FliP, FlhF,

316 FlgN, FliS, FlhB, FliO, FliQ ( 16.0\%); flagellar M-ring protein, FliF (5.6\%); and flagellar

317 regulatory protein, FleQ $(5.3 \%)$ were predominantly associated with cell motility

318 (Supplementary Data 2). Twenty six functional genes encoding different proteins were found

319 to be associated with bacterial chemotaxis (Supplementary Fig. 4, Supplementary Data 2), of

320 them, methyl-accepting chemotaxis protein, mcp (44.2\%); chemotaxis family proteins of

321 bacterial two component system, CheV, CheA, CheB, CheBR, CheY ( 15.0\%); aerotaxis

322 receptor, Aer (7.5\%); MotB (5.2\%) and MotA (3.1\%) were most abundant among these CM

323 microbiotas (Supplementary Data 2). To explore the role of regulation and cell signaling

324 mechanisms in mammary gland pathogenesis, using the SEED subsystem module of MR 
325 analysis, we found two-component regulatory systems BarA-UvrYBarA-UvrY(sirA) as the

326 most abundant virulence regulatory gene (84.1\%) in CM microbiomes (Supplementary Data

327 2). Another regulatory and cell signaling gene, endoplasmic reticulum chaperon grp78 (BiP)

328 was also found as the single most abundant (93.8\%) gene in proteolytic pathways of the CM

329 associated bacterial strains (Supplementary Fig. 5, Supplementary Data 2). A deeper look at

330 microbial genes associated with phages-prophages, transposable elements and plasmids

331 revealed that pathogenicity islands related proteins such as methionine-ABC transporter

332 substrate-binding protein (33.8\%), GMP synthase (27.7\%), tmRNA-binding protein; SmpB

333 (16.0\%), heat shock protein 60; GroEL (16.0\%) and SSU ribosomal protein; S18p (6.1\%)

334 were predominantly abundant among the CM pathogens (Supplementary Data 2). The

335 SEED module analysis also enabled us to identify 28 different protein functions associated

336 with oxidative stress responses among the CM microbiomes which were mostly represented

337 by catalase related proteins (26.7\%), $\mathrm{Cu}-\mathrm{Zn}-\mathrm{Fe}-\mathrm{Mn}$ mediated superoxide dismutases (12.7\%),

$338 \mathrm{H}_{2} \mathrm{O}_{2}$-inducible genes activator (7.8\%) and paraquat-inducible protein $\mathrm{B}$ (7.3\%) (Fig. 9,

339 Supplementary Data 2).

\section{Discussion}

342 Previously, we reported that bovine CM milk microbiomes is a reservoir of diverse groups of

343 resistiome (antibiotics and metal resistance, biofilm formation and quorum sensing genes)

344 with functional biases in metabolism, bacterial chemotaxis, virulence regulation, compared to

345 healthy milk microbiomes ${ }^{2}$. In this study, we employed a combination of both in silico

346 (whole metagenome sequencing, WMS) and in vitro (culture-based) approaches to elucidate

347 the resistome diversity in CM associated microbiomes. Recently, the WMS and other high-

348 throughput sequencing (targeted amplicon) studies have provided new insights into the

349 structure, function and dynamics of bovine $\mathrm{CM}$ milk ${ }^{2-4,12}$ and human lactational mastitis 
350 milk ${ }^{24}$ microbiomes. Our present findings are sufficiently enriched in taxonomic resolution

351 and predicted protein functions, and corroborates to the findings of several previous studies ${ }^{2-}$

$352{ }^{4,24}$. The occurrence of bovine mastitis could be affected by cattle breeds ${ }^{12,15,16}$, and the 353 diversity of CM-causing pathogens is associated with broad range of host-defense 354 mechanisms as part of its immunological arsenal ${ }^{25,26}$. We found significant differences in 355 taxonomic diversity and abundances among the CM microbiomes of four dairy breeds. The 356 XHF cows suffering from $\mathrm{CM}$ had higher microbial diversity at strain-level, and a significant 357 proportion of the microbiota found to be shared with that of the other three breeds (LZ, SW 358 and RCC). Consistent with the results of earlier studies ${ }^{12,15,16,26}$, the taxonomic profile of the 359 CM microbiomes found in four breeds of cows were dominated by phyla Proteobacteria, 360 Bacteroidetes, Firmicutes, Actinobacteria and Fusobacteria. This breed specific variation in 361 taxonomic richness and diversity of microbiome, especially in XHF and LZ cows, could be 362 associated with their increased disease resistance or immune response ${ }^{12,15,16}$ and rumen 363 microbial features (e.g., taxa, diversity indices, functional categories, and genes) ${ }^{26}$. However, 364 further investigations will be necessary to evaluate the real effect of breed specific bacteria on 365 cow mammary gland diseases.

366 Based on previously available culture-based reports on dairy animal mastitis 367 pathogens in Bangladesh ${ }^{6,27}$ and other countries ${ }^{1,7-9}$, we identified six aerobic bacteria $(S$. 368 aureus, E. coli, Klebsiella, Enterobacter, Bacillus and Shigella) through 16S ribosomal RNA 369 (16S rRNA) gene sequencing and phenotypic characterizations, and these findings are in line 370 with the taxonomic signature of WMS. Recent understanding regarding evolutionary 371 relationships of major $\mathrm{CM}$ causing bacteria are primarily based on 16S rRNA gene 372 phylogenetic identification along with a few individual gene or protein sequences ${ }^{28}$, which 373 often produces conflicting phylogenies. This study also explored that the prevalence of CM 374 milk pathogens could vary according to geographical locations and farming (semi-intensive 
375 to intensive grazing system in SER, semi-intensive to free-range grazing systems in CR)

376 systems ${ }^{1}$. These differences may imply that the etiology of bovine CM in Bangladesh could

377 be related to the breed/host genetic factors ${ }^{12,15,16,26}$, types of feeding and farm locations and

378 types $^{1}$, and types of antibiotics and/or metals used for treatment or other factors as have been

379 described in other countries ${ }^{1,8,9}$.

380 Data presented here coupled with the data reported in our earlier study ${ }^{2}$ provides important

381 insights into the diversity of resistomes in CM microbiomes. Our results are concordant with

382 MDR bacteria reported elsewhere from the milk of clinically infected cows ${ }^{8,15,21}$, buffalo

383 cows $^{9}$ and humans ${ }^{11,29}$. Our findings linked multidrug resistance to efflux pumps (MREP),

384 CmeABC operon, mdt ABCD cluster, BlaR1 family, methicillin resistance in Staphylococcus

385 (MRS), resistance to fluoroquinolones (RFL), and multiple metals resistance to CZCR and

386 AR as the predominantly abundant antibiotics and toxic compounds resistance (RATC)

387 functional groups in $\mathrm{CM}$ microbiomes suggesting that bovine $\mathrm{CM}$ milk microbiome

388 constitutes a good reservoir for antimicrobial resistance ${ }^{2,11,29-33}$. It has been reported that

389 efflux pumps regulated by two-component systems in several pathogens, including $A$.

390 baumannii and K. pneumonia, provide multidrug resistance, which may limit the treatment

391 options against bacterial infections of the mammary glands ${ }^{31,32}$. Relative over-expression of

392 efflux pumps enhances the resistances to antimicrobials by reducing the accumulation of

393 antibiotics inside of the bacterial cells and providing sufficient time for the bacteria to adapt

394 to the antibiotics (slow phase antibiotic efflux), and through mutations or alteration of

395 antibiotic targets ${ }^{31,33}$. The CmeABC operon is highly potent against multiple antibiotics,

396 promotes the emergence of ARGs, and confers exceedingly high-level resistance to

397 fluoroquinolones ${ }^{33}$. Therefore, multidrug resistance to efflux pumps and multiple heavy

398 metals resistance represented ubiquitous resistance mechanisms among CM microbiomes,

399 which might be associated with unethical overuse of antibiotics in dairy animals ${ }^{8,9,15,19-21}$ and 
400 extensive application of toxic chemicals and metals in agricultural use $\mathrm{e}^{1,22,34}$ or might have a

401 function in the gut microbiome that is still unknown ${ }^{13,29,35,36}$. The RATC genes detected in

402 this study are of particular interest because there is concern that the use of this class of

403 antibiotics or metals in veterinary medicine, particularly for food animals, may contribute to

404 the development of resistance to this class of antimicrobial options in human ${ }^{29,35}$.

405 In-vitro antibiogram of this study report higher prevalence of resistance to tetracyclines

406 (tetracycline and doxycycline), quinolones (nalidixic acid), penicillins (ampicillin) and

407 phenols (chloramphenicol), similar findings were observed in previous studies on bovine

408 mastitis $^{8,9}$. The AMR profile of bovine CM pathogens for different antimicrobials could vary

409 according to the type and origin of bacteria ${ }^{8-10}$ and host-population such as bovine $e^{8,21}$ and

410 bubaline cows ${ }^{9}$. Consistent with bacterial needs, heavy metals can be transformed (e.g.,

411 oxidized, reduced, methylated, or complexed) and used as a source of energy, terminal

412 electron acceptors, or enzyme structural elements ${ }^{34}$. The highest abundance of CZCR genes

413 among $\mathrm{CM}$ pathogens is mainly due to the presence of $\mathrm{Co}, \mathrm{Zn}$, and $\mathrm{Cd}$ detoxification

414 systems $^{34}$. Although the knowledge on uncontrolled spread of ARGs in bovine mastitis

415 pathogens ${ }^{8}$ are increasing, but information on toxic compounds or heavy metal resistance is

416 yet unavailable. In this study, heavy metals $(\mathrm{Cr}, \mathrm{Co}, \mathrm{Ni}$ and $\mathrm{Cu})$ tested for antibacterial

417 sensitivity showed good efficacy, although knowledge on their mode of action is limited.

418 Thus, with the increase of MDR bacteria in CM, it is imperative that new biocidal and

419 antimicrobial formulations are needed. The MIC and MBC tested metals revealed e $\square$ ective

420 antimicrobial efficacies against a wide range of AMR pathogens ${ }^{1,22,36}$. We found that $\mathrm{Cr}$ and

421 Co compounds had the highest antimicrobial efficacy (MIC) against all of the tested bacteria

422 supported by several previous studies ${ }^{22,37}$. Furthermore, our present findings also suggested

423 that the host genetic component in cattle breeds can significantly regulate the composition of

424 the milk microbiome ${ }^{12,15,16}$, albeit not associated with resistomes profiles. Biofilm formation 
425 is an important virulence factor that may result in recurrent or persistent udder infections ${ }^{38}$

426 and treatment failure through increased resistance to antibiotics and protection against host

427 defences $^{39}$. The relative overexpression of genes encoding lsrACDBFGE operon, biofilm

428 adhesion biosynthesis (BAB), protein $\mathrm{YjgK}_{\mathrm{K}}$ cluster and quorum sensing: autoinducer-2

429 synthesis (QSAU2) in CM microbiomes is in accordance with several earlier reports ${ }^{2,39,40}$. In

430 this study, the relative abundance of the predicted proteins for biofilms and quorum sensing

431 (BF-QS) varied significantly among the selected six bacterial taxa. The BF and QS can be

432 the strain specific or genetically linked traits, representing a selective advantage in

433 pathogenesis of bovine $\mathrm{CM}^{40}$. $\mathrm{BF}$ can enhance proliferation of reactive oxygen and nitrogen

434 species $^{34}$ that can survive antibiotic treatment leading to the transfer of $\mathrm{ARGs}^{41}$. In this

435 study, overall, $76.2 \%$ of the isolates were detected as biofilm formers, and their ability to

436 producing biofilm varied significantly ${ }^{38,39}$. A large number of food spoilage and/or

437 pathogenic bacteria, including Enterococcus faecalis, Enterobacter spp., Pseudomonas spp.,

438 Klebsiella spp., S. aureus, E. coli, B. cereus, and others, have already been associated with

439 biofilms from dairy niches ${ }^{22,23,38-40}$, which supports our current findings.

440 Bacterial chemotaxis mediated by flagellar activities ${ }^{41}$, and the flagella mediated

441 virulence factors are found in many pathogenic species of bovine CM microbiomes, making

442 them a potential target for new antibacterial therapeutics ${ }^{41}$. The intra- and interspecies cell-to-

443 cell communication in bovine CM microbiomes were associated with 26 different genes,

444 which might have vital roles in the early phase of mastitis for attachment to or entry into the

445 udder tissues and virulence regulation ${ }^{42}$ and bacterial colonization in mammary tissues like

446 other suitable sites $^{43}$. The cheA-cheY two-component system mediated bacterial chemotaxis

447 also facilitates the initial contact of bacteria with mammary gland epithelial cells and

448 contribute to effective invasion ${ }^{44}$. The two-component signal transduction system BarA-UvrY

449 regulates metabolism, motility, biofilm formation, stress resistance, virulence and quorum 
450 sensing in $\mathrm{CM}$ pathogens by activating the transcription of genes for regulatory small

$451 \mathrm{RNAs}^{45}$. The up-regulation of genes coding for proteolytic activity, grp78 (BiP) during

452 host-pathogen interactions in $\mathrm{CM}$ is associated with endoplasmic reticulum (ER) stress

453 which further triggers proteolytic activities to initiate the mechanism of pathogenesis and

454 cell death $^{46}$. Catalase activity is a marker of bovine mastitis, which plays a central role in

455 milk redox control and increases markedly during the pathophysiology of bovine $\mathrm{CM}^{47}$. Our

456 present findings corroborated with previous reports ${ }^{47,48}$ that an elevated oxidative stress

457 mediated by catalase activity might have originated either from the mammary gland and/or

458 bacterial cells. During the pathogenesis of bovine mammary gland, bacteria are not rapidly

459 killed by the phagocytic activity of bovine macrophages; rather, they survive within

460 macrophages during prolonged infection due to secretion of catalase and superoxide

461 dismutases, which by degrading $\mathrm{H}_{2} \mathrm{O}_{2}$ inhibit ROS mediated killing mechanism of the $462 \operatorname{host}^{47,48}$.

463

464 Conclusions

465 The bovine CM milk microbiomes harbor diverse groups of resistomes and other virulence

466 factors. The diversity of resistomes positively correlated with the diversity of the microbial

467 communities. The efflux pumps mediated multidrug resistance, methicillin, fluoroquinolones

468 and beta-lactamase resistance, and multiple heavy metals (e.g., cobalt, zinc, cadmium, arsenic

469 and chromium) resistance were the predominating in CM pathogens. Cattle breed is also a

470 predominant factor for $\mathrm{CM}$ associated microbiome diversity, although resistome diversity

471 does not affected by the breed specific microbiome signature. In bovine CM, biofilms may

472 involve in colonizing the pathogens to udder tissues and teat canals, have an important role in

473 antimicrobials resistance, resistant marker transfer and other virulence expression.

474 Furthermore, flagellar movement and chemotaxis, regulation and cell signaling, phages- 
475 prophages, transposable elements, plasmids and oxidative stress had association with the

476 pathophysiology of bovine CM. Therefore, accurate and timely identification of CM

477 microbiome and its associated resistomes along with selection of proper therapeutic regimens

478 will help improve the antimicrobials stewardship for prevention and control of bovine CM in

479 Bangladesh.

\section{$481 \quad$ Methods}

\section{Screening for clinical mastitis (CM) and sampling}

483 We screened 260 quarter milk samples collected from 260 clinical mastitis (CM) affected 484 cows belonging to 50 smallholding dairy farms in two geographical regions of Bangladesh 485 (central region, $C R=160$; southeastern region, $S E R=100$ ) (Supplementary Fig. 1). The cows 486 represented four different breeds, including local zebu (LZ), red Chattogram cattle (RCC), 487 Sahiwal (SW), and crossbred Holstein Friesian (XHF) at their early stage of lactation (within 488 10-40 days post-calving). A screening test for $\mathrm{CM}$ was conducted using the California 489 Mastitis Test $\left(\mathrm{CMT}^{\circledR}\right.$, Original Schalm reagent, ThechniVet, USA) ${ }^{49}$. Approximately 15-20 490 ml of milk from each cow was collected under aseptic conditions in a sterile falcon tube 491 during the morning milking (8.00-10.00 am), and kept on ice (at $4^{\circ} \mathrm{C}$ ) for transport to the 492 laboratory for subsequent processing.

\section{Metagenomic DNA extraction and sequencing}

494 Genomic DNA (gDNA) from 25 randomly selected CM samples was extracted by an 495 automated Maxwell 16 DNA extraction platform using blood DNA purification kits 496 (Promega, UK) following previously described protocols ${ }^{2}$. DNA quantity and purity were 497 determined with NanoDrop (ThermoFisher, USA) by measuring 260/280 absorbance ratios. 498 Sequencing libraries were prepared with Nextera XT DNA Library Preparation Kit ${ }^{50}$ and 499 paired-end $(2 \times 150 \mathrm{bp})$ sequencing was performed on a NextSeq 500 machine (Illumina Inc., 
500 USA) at the George Washington University Genomics Core facility. Our metagenomic DNA

501 yielded 596.74 million reads with an average of 23.87 million (maximum=39.75 million,

502 minimum=8.89 million) reads per sample (Supplementary Data 1).

\section{Sequence reads preprocessing}

504 The resulting FASTQ files were concatenated and filtered through BBDuk ${ }^{2}$ (with options

$505 \mathrm{k}=21, \operatorname{mink}=6, \mathrm{ktrim}=\mathrm{r}, \mathrm{ftm}=5$, qtrim=rl, trimq=20, minlen=30, overwrite=true) to remove

506 Illumina adapters, known Illumina artifacts, and phiX. Any sequence below these thresholds

507 or reads containing more than one ' $\mathrm{N}$ ' were discarded. On average, 21.13 million reads per

508 sample (maximum=36.89 million, minimum=4.71 million) passed the quality control step

509 (Supplementary Data 1).

\section{Microbiome diversity and community analysis}

511 The shotgun whole metagenome sequencing (WMS) data were analyzed using both mapping-

512 based and assembly-based hybrid methods of PathoScope 2.0 (PS) ${ }^{51}$ and MG-RAST (MR),

513 respectively ${ }^{52}$. In PS analysis, a 'target' genome library was constructed containing all

514 bacterial sequences from the NCBI Database using the PathoLib module ${ }^{51}$. The reads were

515 then aligned against the target libraries using the very sensitive Bowtie 2 algorithm ${ }^{53}$ and

516 filtered to remove the reads aligned with the cattle genome (bosTau8) and human genome

517 (hg38) as implemented in PathoMap (-very-sensitive-local -k 100 --score-min L,20,1.0).

518 Finally, the PathoID ${ }^{54}$ module was applied to obtain accurate read counts for downstream

519 analysis. In these samples, 17.20 million reads (4.3\% of total reads) mapped to the target

520 reference genome libraries after filtering the cow and human genome (Supplementary Data

521 1). The raw sequences were simultaneously uploaded to the MR server (release 4.0) with

522 proper embedded metadata and were subjected to the quality filter containing dereplication

523 and removal of host DNA by screening for taxonomic and functional assignment. Alpha

524 diversity (diversity within samples) was estimated using the observed species, Chao1, ACE, 
525 Shannon, Simpson and Fisher diversity indices ${ }^{55}$ for both PS and MR read assignments and

526 counts. To visualize differences in bacterial diversity, a principal coordinate analysis (PCoA)

527 was performed based on weighted-UniFrac distances (for PS data) through Phyloseq R

528 package (version 3.5.1) ${ }^{56}$ and Bray-Curtis dissimilarity matrix ${ }^{57}$ (for MR data). We have also

529 used OmicCircos (version 3.9) ${ }^{58}$ which is an R package based on python script for circular

530 visualization of both microbiome diversity and resistance to antibiotics and toxic compounds

531 (RATC) functional groups found in MR data for respective four breeds of CM cows.

\section{In vitro identification of bacteria}

533 Collected CM milk samples $(\mathrm{n}=260)$ were subjected to selective isolation and identification

534 of S. aureus, E. coli, Klebsiella, Enterobacter, Shigella and Bacillus species according to

535 previously described microbiological methods ${ }^{1,6-9}$. The pathogens were identified based on

536 their colony morphology, hemolytic patterns on blood agar and Gram-staining ${ }^{8}$. Gram-

537 positive bacteria were further confirmed based on their biochemical characteristics in indole,

538 methyl red, Voges-Proskauer (VP), catalase, oxidase, urease and triple sugar iron (TSI) tests,

539 and growth on mannitol salt agar. Gram-negative bacteria were confirmed based on the

540 results of indole, methyl red, citrate (IMViC) tests and lactose fermentation on Mac agar ${ }^{9,40}$.

541 Finally, all isolates were stored at $-80{ }^{\circ} \mathrm{C}$ for further genomic identification.

\section{PCR amplification and ribosomal (16S rRNA) gene sequencing}

543 Genomic DNA of probable S. aureus, E. coli, Klebsiella, Enterobacter, Shigella, and Bacillus

544 species was extracted from overnight cultures using the boiled method ${ }^{59}$. The quantity and

545 purity of the extracted DNA was determined as mentioned before. The 16S rRNA gene was

546 amplified using universal primers 27F (5'-AGAGTTTGATCCTGGCTCAG-3') and U1492R

547 (5'-CTACGGCTACCTTGTTACGA-3' $)^{60}$. Agarose gel electrophoresis $(1.2 \% \mathrm{wt} / \mathrm{vol})$ was

548 used to verify the presence of PCR products. DNA sequencing was carried out at First Base

549 Laboratories Sdn Bhd (Malaysia) using Applied Biosystems highest capacity-based genetic 
550 analyzer (ABI PRISM ${ }^{\circledR} 377$ DNA Sequencer) platforms with the BigDye ${ }^{\circledR}$ Terminator v3.1

551 cycle sequencing kit chemistry ${ }^{61}$.

\section{Phylogenetic analysis of the microbial communities}

553 Taxonomic abundance of the WMS data was determined by applying the "Best Hit

554 Classification" option in PS pipeline using the NCBI database as a reference with the 555 following settings: maximum e-value of $1 \times 10^{-30}$; minimum identity of $95 \%$ for bacteria, and a 556 minimum alignment length of 20 as the set parameters. A phylogenetic tree consisting of the 557 top 200 abundant bacterial strains identified through PS analysis from the WMS reads of the $55825 \mathrm{CM}$ samples with $>90 \%$ taxonomic identity was constructed using maximum-likelihood 559 method in Clustal W (version 2.1) ${ }^{61}$ and visualized using interactive Tree Of Life (iTOL) ${ }^{62}$.

560 Another, phylogenetic tree consisting of 40 strains correspondent to in vitro examined six

$561 \mathrm{CM}$ bacteria found in $260 \mathrm{CM}$ samples with $>90 \%$ taxonomic identity was also constructed 562 using same methods. Using Molecular Evolutionary Genetics Analysis (MEGA) version 7.0

563 for bigger datasets ${ }^{63}$, the 16S rRNA gene sequences, amplified from all individual bacterial 564 isolates, were aligned with each other and with relevant reference sequences obtained from 565 the NCBI Database, and a maximum-likelihood tree was generated using these 16S rRNA 566 gene sequences ${ }^{63}$. The percentage of replicate trees in which the associated taxa clustered 567 together in the bootstrap test (1000 replicates) is shown next to the branches ${ }^{64}$.

\section{Antimicrobial susceptibility testing}

569 The in vitro antibiogram profile of $221 \mathrm{CM}$ isolates was determined using the disk diffusion 570 method following the Clinical Laboratory Standards Institute ${ }^{65}$ guidelines. Antibiotics were 571 selected for susceptibility testing corresponding to a panel of antimicrobial agents (Oxoid ${ }^{\mathrm{TM}}$,

572 Thermo Scientific, UK) of interest to the dairy industry and public health in Bangladesh. The 573 selected groups of antibiotics were commonly used in treating CM by the dairy farmers and 574 included penicillins (ampicillin, $10 \mu \mathrm{g} / \mathrm{mL}$ ), tetracyclines (doxycycline, $30 \mu \mathrm{g} / \mathrm{mL}$; 
575 tetracycline, $30 \mu \mathrm{g} / \mathrm{ML}$ ), nitrofurans (nitrofurantoin, $300 \mu \mathrm{g} / \mathrm{mL}$ ), quinolones (ciprofloxacin,

$57610 \mu \mathrm{g} / \mathrm{mL}$; nalidixic acid, $30 \mu \mathrm{g} / \mathrm{mL}$ ), cephalosporins (cefoxitin, $30 \mu \mathrm{g} / \mathrm{mL}$ ), penems

577 (imipenem, $10 \mu \mathrm{g} / \mathrm{mL}$ ), phenols (chloramphenicol, $30 \mu \mathrm{g} / \mathrm{mL}$ ), aminoglycosides (gentamycin,

$57810 \mu \mathrm{g} / \mathrm{mL}$; vancomycin, $30 \mu \mathrm{g} / \mathrm{mL}$ ), macrolides (erythromycin, $15 \mu \mathrm{g} / \mathrm{mL}$ ). Resistance was

579 defined according to CLSI (2017) with slight modifications ${ }^{8,9}$.

\section{$580 \quad$ Metal susceptibility testing}

581 The antibacterial effect of heavy metals was evaluated in vitro for the isolated pathogens

582 using both agar well diffusion and tube dilution methods ${ }^{1,22}$. Five heavy metals such as 583 copper $(\mathrm{Cu})$, zinc $(\mathrm{Zn})$, chromium $(\mathrm{Cr})$, nickel $(\mathrm{Ni})$, and cobalt $(\mathrm{Co})$ were used as salts:

$584 \mathrm{CuSO} 4.5 \mathrm{H} 2 \mathrm{O}, \mathrm{ZnSO} 4.7 \mathrm{H} 2 \mathrm{O}, \mathrm{K} 2 \mathrm{Cr} 2 \mathrm{O} 7, \mathrm{NiCl} 2$, and $\mathrm{CoCl} 2.6 \mathrm{H} 2 \mathrm{O}$, respectively to study the

585 level of zone of inhibition (ZOI). Briefly, pure culture of the isolated pathogens from NA

586 plates were sub-cultured into Mueller-Hinton agar (Oxoid ${ }^{\mathrm{TM}}$, UK) plates, and five $7 \mathrm{~mm}$

587 wells were made, one in the center of the plate and the other four about $20 \mathrm{~mm}$ away from the

588 center. Varying concentrations of the metal solutions were prepared $(2,4,8,16,32,48$ and

$58964 \mu \mathrm{g} / \mathrm{mL}$ ) and $100 \mu \mathrm{l}$ of prepared solution was inoculated into the central well of $1 \mathrm{~cm}$ in

590 diameter. The plates were incubated at $37{ }^{\circ} \mathrm{C}$ for $24 \mathrm{~h}$ to allow diffusion of the metal into the

591 agar, and the antibacterial activity was determined by measuring the diameter of ZOI in

$592 \mathrm{~mm}^{12}$. After investigating the resistance profile of the isolates at different concentrations, the

593 minimal inhibitory concentration (MIC) of the metals was determined by the tube dilution

594 method by gradually increasing or decreasing the heavy metal concentrations ${ }^{1}$. Finally,

595 growth of bacterial colonies was observed and the concentration that showed no growth was 596 considered as the minimum bactericidal concentration (MBC) ${ }^{1}$.

\section{Biofilm assay and microscopy}

598 Microtiter plate assays were performed to screen for biofilm formation (BF) ability of 80

599 randomly selected isolates using standard protocols ${ }^{22,23,38,39}$. We quantified the absorbance of 
600 solubilized crystal violet (CV), in a plate reader at $600 \mathrm{~nm}$ using $30 \%$ acetic acid in water as

601 the blank and TSB as negative control. The solution was removed, and the absorbance 602 measured at optical density-590 (OD590) $(n=3)$. To determine BF ability of strains, cut-off

603 OD (ODc) was defined as three standard deviations above the mean OD of the negative 604 control. Strains were classified as: non-biofilm formers, NBF (OD $\leq$ ODc); weak biofilm 605 formers, WBF (ODc < OD $\leq 2 \times$ ODc); moderate biofilm formers, $\mathrm{MBF}(2 \times \mathrm{ODc}<\mathrm{OD} \leq 4$ $606 \mathrm{x}$ ODc) and strong biofilm formers, $\mathrm{SBF}(\mathrm{OD}>4 \mathrm{x} \text { ODc })^{22,39}$. In this study, the ODc value 607 was set as 0.045 and the mean OD of the negative control was $0.039 \pm 0.002^{22}$. The biofilms 608 were then visualized using 5\% TSB as nutrient rich media and FilmTracer ${ }^{\mathrm{TM}}$ LIVE/DEAD ${ }^{\circledR}$ 609 Biofilm Viability Kit as staining materials under Olympus BX51 upright microscope at 40X 610 objective, and finally images were collected using Olympus DP73 camera through cellSens

611 entry software (Olympus Corporation, Japan) and visualized using image $\mathbf{J}$ software ${ }^{39}$. As a 612 negative control, we used E. coli DH5 alpha for all the in vitro resistome (antimicrobial and 613 metal susceptibility tests and biofilm assays) analysis tests.

\section{Microbial functional analysis}

615 Metagenomic functional composition was based on the gene families from different levels of 616 SEED module and the Kyoto Encyclopedia of Genes and Genomes (KEGG) database ${ }^{66}$ using 617 the MG-RAST 4.1 (MR) pipeline ${ }^{52}$. We observed significant differences (Kruskal-Wallis 618 test, $p=0.001$ ) in the relative abundance of genes coding for RATC and microbial functional 619 genomic potentials in four cattle breeds.

\section{Statistical analysis}

621 The characteristics of breeds of the cows with CM were compared using a Kruskal-Wallis 622 test for quantitative variables ${ }^{2}$. The Shapiro-Wilk test was used to check normality of the 623 data, and the non-parametric test Kruskal-Wallis rank sum test was used to evaluate 624 differences in the relative abundance of bacterial taxa at strain level according to breed 
625 groups ${ }^{12,15,16}$. The statistical analyses for the MR data were initially performed by embedded

626 calls to statistical tests in the pipeline and validated further using IBM SPSS (SPSS, Version

627 23.0, IBM Corp., NY USA) using the above mentioned tests. For the functional abundance

628 profiling, the statistical (Kruskal-Wallis test and Pearson correlation) tests were applied at

629 different KEGG and SEED subsystem levels in the MR pipeline ${ }^{52}$. To evaluate the significant

630 relationships between identified bacterial species and the study region, we used the two-

631 sample proportions test using SPSS. Results were considered statistically significant when

$632 p<0.05$ and highly significant when $p<0.01$. Mean values were used to compare the

633 antimicrobial efficacy results of the tested antibiotics and heavy metals at varying

634 concentrations. Standard error means were calculated to analyze the distributions of the data

635 from the mean value and confidence intervals of $95 \%$ were calculated for the MIC and MBC

636 tests results to plot error bars ${ }^{22,39}$. We also performed Pearson correlation tests to test for

637 relationships between taxonomic abundance of the pathogens and antimicrobial resistance

638 both for cultural and metagenomic data. A post hoc Bonferroni test was used to compare the

639 biofilm OD600 mean values ${ }^{22,39}$.

\section{Acknowledgements}

641 The authors thank Stephanie Warnken, $\mathrm{PhD}$ student at the Computational Biology Institute,

642 Milken Institute School of Public Health, The George Washington University, USA for their

643 technical support in learning basic bioinformatics operations. We also acknowledge the 644 cooperation of the dairy farmers for allowing us to conduct the study in their farms.

\section{Author contributions}

646 M.N.H., M. S., A.I. and M.A.H. conceived and designed the overall study. M.N.H. surveyed 647 and collected all the field samples. M.N.H., R.A.C., K.M.G, O.S. and O.K.I. carried out 648 laboratory works including DNA extractions and sequencing, microbiological (cultural, 649 biochemical) examinations, antimicrobial (antibiotics, metals) sensitivity tests and biofilm 
650 assays. M.A.H. and K.A.C. contributed chemicals and reagents. M.N.H. and A.I. conceived,

651 designed and executed the bioinformatics analysis. M.N.H. interpreted the results and drafted

652 the manuscript. M.S., K.A.C. and M.A.H contributed intellectually to the interpretation and

653 presentation of the results. Finally, all authors have approved the manuscript for submission.

655 Funding

656 The Bangladesh Bureau of Educational Information and Statistics (BANBEIS), Ministry of

657 Education, Government of the People's Republic of Bangladesh (Grant No. LS2017313)

658 supported this work.

659 Supplementary Information

660 Supplementary information supporting the findings of the study are available in this article as

661 Supplementary Data files, or from the corresponding author on request.

\section{Conflict of Interest Statement}

663 The authors declare no competing interests.

\section{Data availability}

665 The sequence data reported in this paper have been deposited in the NCBI database 666 (BioProject PRJNA529353 for metagenome sequences, NCBI accession number: MN 667 620423-MN 620430 for 16S rRNA gene sequences) and are available from the corresponding 668 author upon reasonable request. 
675

676

677

678

679

680

681

682

683

684

685

686

687

688

689

690

691

692

693

694

695

696

697

698

699

\section{References}

1. Reyes-Jara, A., Cordero, N., Aguirre, J., Troncoso, M. \& Figueroa, G. Antibacterial Effect of Copper on Microorganisms Isolated from Bovine Mastitis. Front. Microbiol. 7, 626 (2016).

2. Hoque, M. N. et al. Metagenomic deep sequencing reveals association of microbiome signature with functional biases in bovine mastitis. Sci. Rep. 9,13536 (2019).

3. Oikonomou, G. et al. Microbiota of cow's milk; distinguishing healthy, subclinically and clinically diseased quarters. PLoS One 9(1), e85904 (2014).

4. Falentin, H. et al. Bovine Teat Microbiome Analysis Revealed Reduced Alpha Diversity and Significant Changes in Taxonomic Profiles in Quarters with a History of Mastitis. Front. Microbiol. 7 (2016).

5. Abebe, R., Hatiya, H., Abera, M., Megersa, B. \& Asmare, K. Bovine mastitis: prevalence, risk factors and isolation of Staphylococcus aureus in dairy herds at Hawassa milk shed, South Ethiopia. BMC Vet. Res. 12(1), 270 (2016).

6. Hoque, M. N. et al. Molecular characterization of Staphylococcus aureus strains in bovine mastitis milk in Bangladesh. Int. J. Vet. Sci. Med. 6(1), 53-60 (2018).

7. Gao, J. et al. Incidence of clinical mastitis and distribution of pathogens on large Chinese dairy farms J. Dairy Sci. 100(6), 4797-4806 (2017).

8. Cheng, J. et al. Antimicrobial resistance profiles of 5 common bovine mastitis pathogens in large Chinese dairy herds. J. Dairy Sci. 102, 1-11 (2018).

9. Preethirani, P. L. et al. Isolation, biochemical and molecular identification, and invitro antimicrobial resistance patterns of bacteria isolated from bubaline subclinical mastitis in South India. PLoS One 10(11), e0142717 (2015).

10. Van Boeckel, T. P. et al. Global trends in antimicrobial use in food animals. Proc. Nat. Aca. Sci. 112, 5649-5654 (2015). 
700 11. Baron, S., Diene, S. \& Rolain, J. M. Human microbiomes and antibiotic resistance.

701 Human Microbiome J. 10, 43-52 (2018).

702 12. Cremonesi, P. et al. Milk microbiome diversity and bacterial group prevalence in a 703 comparison between healthy Holstein Friesian and Rendena cows. PLoS One 13(10), $704 \quad \mathrm{e} 0205054(2018)$

705 13. Oniciuc, E. et al. The present and future of Whole Genome Sequencing (WGS) and 706 Whole Metagenome Sequencing (WMS) for surveillance of antimicrobial resistant 707 microorganisms and antimicrobial resistance genes across the food chain. Genes 9(5), $268(2018)$

709 14. Seth, S., Välimäki, N., Kaski, S. \& Honkela, A. Exploration and retrieval of whole710 metagenome sequencing samples. Bioinformatics 30(17), 2471-2479 (2014).

711 15. Curone, G. et al. What we have lost: Mastitis resistance in Holstein Friesians and in a 712 local cattle breed. Res. Vet. Sci. 116, 88-98 (2018).

713 16. Gonzalez-Recio, O., Zubiria, I., García-Rodríguez, A., Hurtado, A. \& Atxaerandio, R. 714 Signs of host genetic regulation in the microbiome composition in 2 dairy breeds: 715 Holstein and Brown Swiss. J. Dairy Sci. 101(3), 2285-2292 (2018).

716 17. Brandt, C. et al. In silico serine $\beta$-lactamases analysis reveals a huge potential 717 resistome in environmental and pathogenic species. Sci. Rep. 7, 43232 (2017).

718 18. D’Costa, V. M. et al. Antibiotic resistance is ancient. Nature 477, 457-461 (2011).

719 19. Weller, C. \& Wu, M. A generation-time effect on the rate of molecular evolution in $720 \quad$ bacteria. Evolution 69, 643-652 (2015).

721 20. Zaheer, R. et al. Comparative diversity of microbiomes and Resistomes in beef 722 feedlots, downstream environments and urban sewage influent. BMC Microbiol. 723 19,197 (2019). 
724 21. Tomazi, T. et al. Molecular characterization and antimicrobial susceptibility pattern of 725 Streptococcus agalactiae isolated from clinical mastitis in dairy cattle. PLoS One $726 \quad$ 13(6), e0199561 (2018).

727 22. Vaidya, M. Y. et al. Antimicrobial efficacy and synergy of metal ions against 728 Enterococcus faecium, Klebsiella pneumoniae and Acinetobacter baumannii in 729 planktonic and biofilm phenotypes. Sci. Rep. 7(1), 5911 (2017).

730 23. Singh, S., Singh, S. K., Chowdhury, I. \& Singh, R. Understanding the mechanism of 731 bacterial biofilms resistance to antimicrobial agents. The Open Microbiol. J. 11, 53 $732 \quad$ (2017).

733 24. Patel, S. H. et al. Culture independent assessment of human milk microbial 734 community in lactational mastitis. Sci. Rep. 7(1), 7804 (2017).

735 25. Thompson-Crispi, K., Atalla, H., Miglior, F. \& Mallard, B. A. Bovine mastitis: 736 Frontiers in immunogenetics. Front. Immunol. 5, 493 (2014).

737 26. Li, F. et al. Host genetics influence the rumen microbiota and heritable rumen 738 microbial features associate with feed efficiency in cattle. Microbiome 7(1), 92 739 (2019).

740 27. Sumon, S. M. M. R., Ehsan, M. A. \& Islam, M. T. Subclinical mastitis in dairy 741 cows: Somatic cell counts and associated bacteria in Mymensingh, Bangladesh. $J$. 742 Bangladesh Agri. Uni. 15(2), 266-271 (2017).

743 28. Naushad, S. et al. Comprehensive phylogenetic analysis of bovine non-aureus 744 staphylococci species based on whole-genome sequencing. Front. Microbiol. 7, 1990 745 (2016).

746 29. Penders, J., Stobberingh, E., Savelkoul, P. \& Wolffs, P. The human microbiome as a 747 reservoir of antimicrobial resistance. Front. Microbiol. 4, 87 (2013). 
30. Kumar, S. \& Varela, M. F. Biochemistry of bacterial multidrug efflux pumps. Int. J. Mol. Sci. 13(4), 4484-4495 (2012).

31. Tiwari, S. et al. Two-Component Signal Transduction Systems of Pathogenic Bacteria As Targets for Antimicrobial Therapy: An Overview. Front. Microbiol. 8, 1878 (2017).

32. Li, X. Z. \& Nikaido, H. Efflux-mediated drug resistance in bacteria: An update. Drugs 69,1555-1623 (2009).

33. Yao, H. et al. Emergence of a potent multidrug efflux pump variant that enhances Campylobacter resistance to multiple antibiotics. mBio 7(5), e01543-16 (2016). involved in self-purification of mine waters contaminated with heavy metals. Front. Microbiol. 7, 1252 (2016).

35. Hu, Y., Yang, X., Lu, N. \& Zhu, B. The abundance of antibiotic resistance genes in

36. Ciesinski, L. et al. High dietary zinc feeding promotes persistence of multi-resistant $E$. coli in the swine gut. PLoS One 13(1), e0191660 (2018). and cobalt (II) complexes: synthesis and biological activity evaluations. Molecules 23(8), 2013 (2018). mastitis infections? Vet. J. 171(3), 398-407 (2006).

39. Schönborn, S. et al. In vitro ability of mastitis causing pathogens to form biofilms. $J$. Dairy Res. 84(2), 198-201 (2017). 
40. Gomes, F, Saavedra, M. J. \& Henriques, M. Bovine mastitis disease/pathogenicity: evidence of the potential role of microbial biofilms. Patho. Dis. 74(3), 1-7 (2016).

41. Duan, Q., Zhou, M., Zhu, L. \& Zhu, G. Flagella and bacterial pathogenicity. J. Basic Microbiol. 53(1), 1-8 (2013).

42. Zatakia, H. M., Arapov, T. D., Meier, V. M. \& Scharf, B. E. Cellular stoichiometry of methyl-accepting chemotaxis proteins in Sinorhizobium meliloti. J. Bacteriol. 200(6), e00614-17 (2018)

43. Matilla, M. A. \& Krell, T. The effect of bacterial chemotaxis on host infection and pathogenicity. FEMS Microbiol. Reviews 42(1), fux052 (2017).

44. Dons, L. et al. Role of flagellin and the two-component CheA/CheY system of Listeria monocytogenes in host cell invasion and virulence. Infection Immunity, 72(6), 3237-3244 (2004).

45. Zere, T. R. et al. Genomic Targets and Features of BarA-UvrY (-SirA) Signal Transduction Systems. PLoS One 10(12), e0145035 (2015).

46. Hirai, K. E. et al. Endoplasmic Reticulum Stress Markers and Their Possible Implications in Leprosy's Pathogenesis. Dis. Markers, 2018.

47. Andrei, S., Matei, S., Rugină, D., Bogdan, L. \& Ştefănuț, C. Interrelationships between the content of oxidative markers, antioxidative status, and somatic cell count in cow's milk. Czech J. Animal Sci., 61(9), 407-413 (2016).

48. Darbaz, I. et al. Evaluation of milk glutathione peroxidase and superoxide dismutase levels in subclinical mastitis in Damascus goats. Turk. J. Vet. Anim. Sci. 43(2), 259263 (2019).

49. Hoque, M. N. et al. Different screening tests and milk somatic cell count for the prevalence of subclinical bovine mastitis in Bangladesh. Trop. Anim. Health Prod. 47(1), 79-86 (2015). 
50. Head, S. R. et al. Library construction for next-generation sequencing: overviews and 798 challenges. Biotechniques 56(2), 61-77 (2014).

51. Hong, C. et al. PathoScope 2.0: a complete computational framework for strain identification in environmental or clinical sequencing samples. Microbiome 2(1), 33(2014).

52. Glass, E. M., Wilkening, J., Wilke, A., Antonopoulos, D. \& Meyer, F. Using the metagenomics RAST server (MG-RAST) for analyzing shotgun metagenomes. Cold

53. Langmead, B., \& Salzberg, S. L. (2012). Fast gapped-read alignment with Bowtie 2. Nat. Methods 9, 357-359 (2012).

54. Francis, O. E. et al. Pathoscope: species identification and strain attribution with unassembled sequencing data. Genome Res. 23(10), 1721-1729 (2013).

56. McMurdie, P. J. \& Susan, H. Phyloseq: an R package for reproducible interactive analysis and graphics of microbiome census data. PLoS One 8(4), e61217 (2013).

813 57. Beck, J., Holloway, J. D. \& Schwanghart, W. Under sampling and the measurement of beta diversity. Methods Ecol. Evol. 4(4), 370-382 (2013).

58. Hu. Y. et al. OmicCircos: a simple-to-use R package for the circular visualization of

818 effect of immunoglobulin G as an inhibitor in real-time PCR for serum samples from 819 patients with brucellosis. Clin. Vaccine Immunol. 15(2), 293-296 (2008). 
820 60. Masomian, M., Rahman, R. N. Z. R. A., Salleh, A. B. \& Basri, M. Analysis of

821 comparative sequence and genomic data to verify phylogenetic relationship and 822 explore a new subfamily of bacterial lipases. PLoS One 11(3), e0149851 (2016).

823 61. Larkin M. A. et al. Clustal W and Clustal X version 2.0. Bioinformatics 23, 2947$8242948(2007)$.

825 62. Letunic, I. \& Bork, P. Interactive Tree Of Lifev2: online annotation and display of 826 phylogenetic trees made easy. Nucleic Acids Res. 39, W475-W478 (2011).

827 63. Kumar, S., Stecher, G. \& Tamura, K. MEGA7: Molecular Evolutionary Genetics 828 Analysis version 7.0 for bigger datasets. Mol. Biol. Evol. 33(7), 1870-1874 (2016).

829 64. Pattengale, N.D., Alipour, M., Bininda-Emonds, O.R., Moret, B.M. \& Stamatakis, A. 830 How many bootstrap replicates are necessary? J. Comput. Biol. 17(3), 337-354 $831 \quad$ (2010).

832 65. CLSI. Performance standards for antimicrobial susceptibility testing; CLSI document 833 M100-S27. Clinical Laboratory Standards Institute (CLSI), Wayne, PA (2017).

834 66. Kanehisa, M., Sato, Y., Furumichi, M., Morishima, K. \& Tanabe, M. New approach 835 for understanding genome variations in KEGG. Nucleic Acids Res. 47(D1), D590836 D595 (2018). 


\section{Resistome diversity in bovine clinical mastitis microbiome, a signature concurrence}

M. Nazmul Hoque, Arif Istiaq, Rebecca A. Clement, Keylie M. Gibson, Otun Saha, Ovinu Kibria Islam, Ruhshan Ahmed Abir, Munawar Sultana, AMAM Zonaed Siddiki, Keith A. Crandall, M. Anwar Hossain
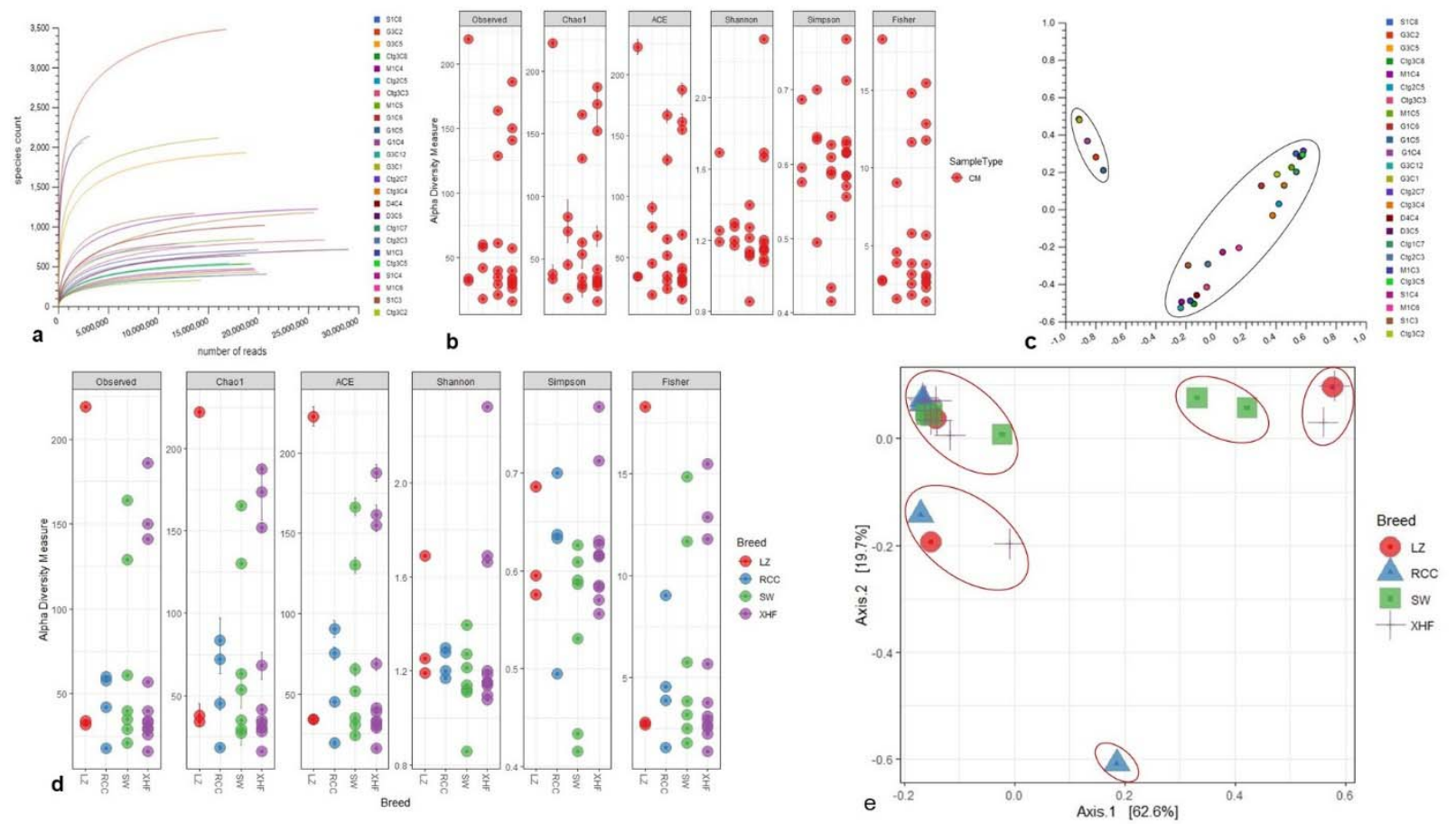

Fig. 1 Bovine clinical mastitis (CM) milk microbiome diversity. a) Rarefaction curves showing the influence of sequencing depth (number of reads per sample, $\mathrm{X}$ axis) on species richness (Y axis) in CM milk samples. The rarefaction curves representing the number of species per sample indicated that the sequencing depth was sufficient enough to fully capture the microbial diversity as existed. b) Alpha diversity measured using the observed species, Chao 1, ACE and Shannon diversity indices through PathoScope (PS) analysis. The observed species richness $\left(\mathrm{P}_{\text {Observed }}=\right.$ 0.511), Chao1 $\left(\mathrm{P}_{\text {Chaol }}=0.081\right)$, ACE $\left(\mathrm{P}_{\mathrm{ACE}}=0.121\right)$, Shannon $\left(\mathrm{P}_{\text {Shannon }}=0.401\right)$, Simpson $\left(\mathrm{P}_{\text {Simpson }}=0.011\right)$ and Fisher $\left(\mathrm{P}_{\text {Fisher }}=0.014\right)$ diversity analyses revealed that microbiome diversity did not vary among the $\mathrm{CM}$ samples. c) Beta diversity (Principal coordinate analysis; PCoA) measured on the Bray-Curtis distance method using MG-RAST tool for CM causing 
microbial communities (genus-level) shows that most of the CM samples clustered together (black circle) indicating no significant diversity differences. d) Alpha diversity measured using species richness $\left(\mathrm{P}_{\text {Observed }}=0.011\right)$, Chao1 $\left(\mathrm{P}_{\text {Chao } 1}=0.001\right), \operatorname{ACE}\left(\mathrm{P}_{\mathrm{ACE}}=0.021\right)$, Shannon $\left(\mathrm{P}_{\text {Shannon }}=0.001\right)$, Simpson $\left(\mathrm{P}_{\text {Simpson }}=0.009\right)$ and Fisher $\left(\mathrm{P}_{\text {Fisher }}=0.023\right)$ diversity matrices on PS data showed significant diversity differences (Kruskal-Wallis test, $p=0.002$ ) within the microbial communities of four breeds (Local Zebu cows, LZ; Red Chattogram cows, RCC; Sahiwal, SW; Holstein Friesian cross, XHF) of cows. e) PCoA plot based on weighted-UniFrac distance method at strain-level microbiome signature of four breeds of cows reveals that the CM samples appear more distantly (red circles) indicating significant group differences $(p=0.001)$. This differences in the microbiome signature associated with $\mathrm{CM}$ in four breeds could be explained by a large percentage of variation in the first (62.6\%) and second (19.7\%) axes. 


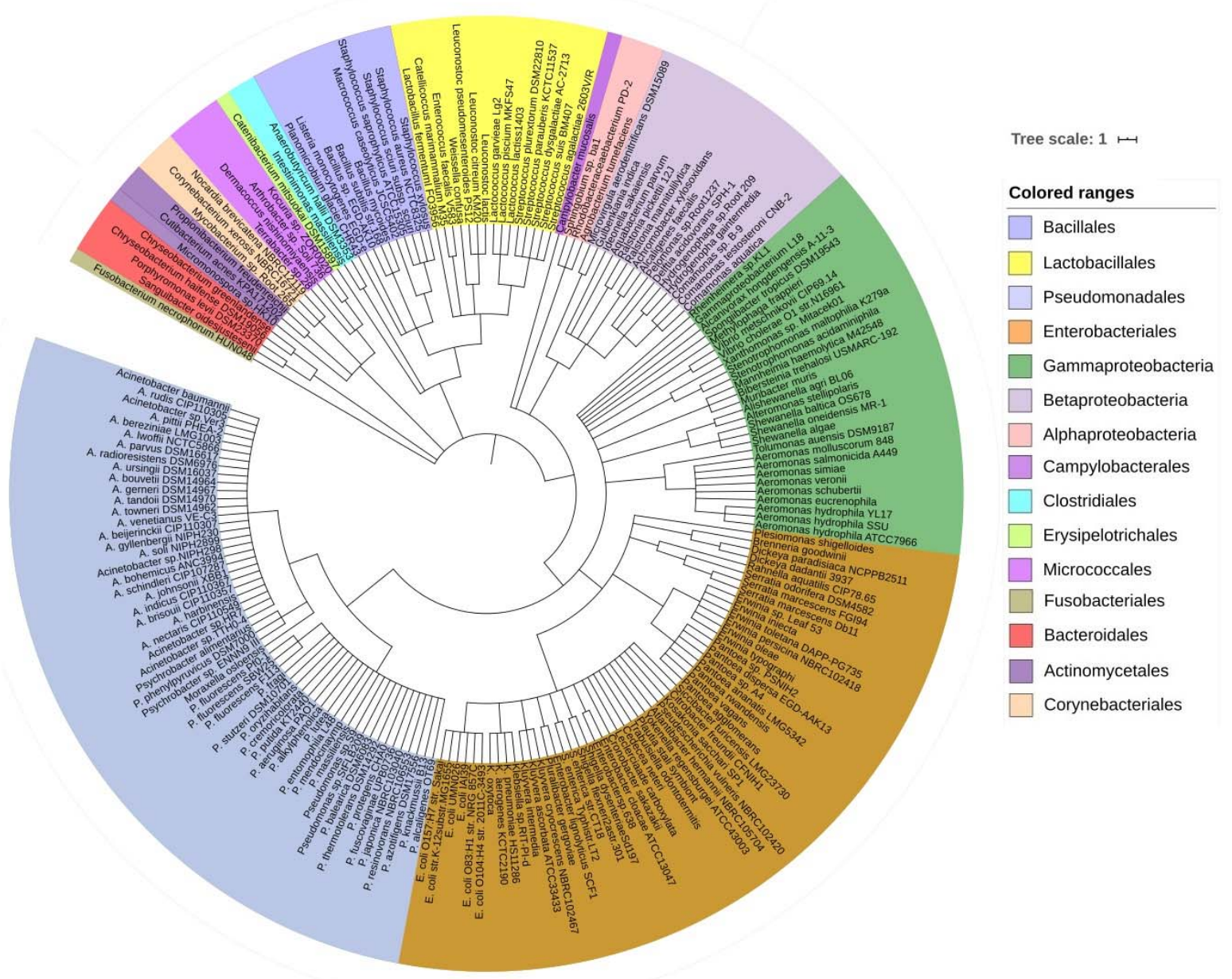

Fig. 2 The strain-level taxonomic profile microbiota associated with bovine clinical mastitis (CM). Taxonomic dendrogram showing the top bacterial microbiome of bovine CM milk. Color ranges identify different strains within the tree. Taxonomic dendrogram was generated with the top 200 abundant unique strains of bacteria in CM milk metagenome based on the maximum likelihood method in Clustal W and displayed with iTOL (interactive Tree Of Life). Each node represents a single strain shared among more than $50 \%$ of the samples at a relative abundance of $>0.0006 \%$ of the total bacterial community. The inner circle represents the root of the microbiome defined as bacteria present in $25 \mathrm{CM}$ milk samples. The outer circle shows the strains and/or species colored by different order of bacteria present in $>80 \%$ of samples. The strains in the phylogenetic tree are also available in Supplementary Data 1. 

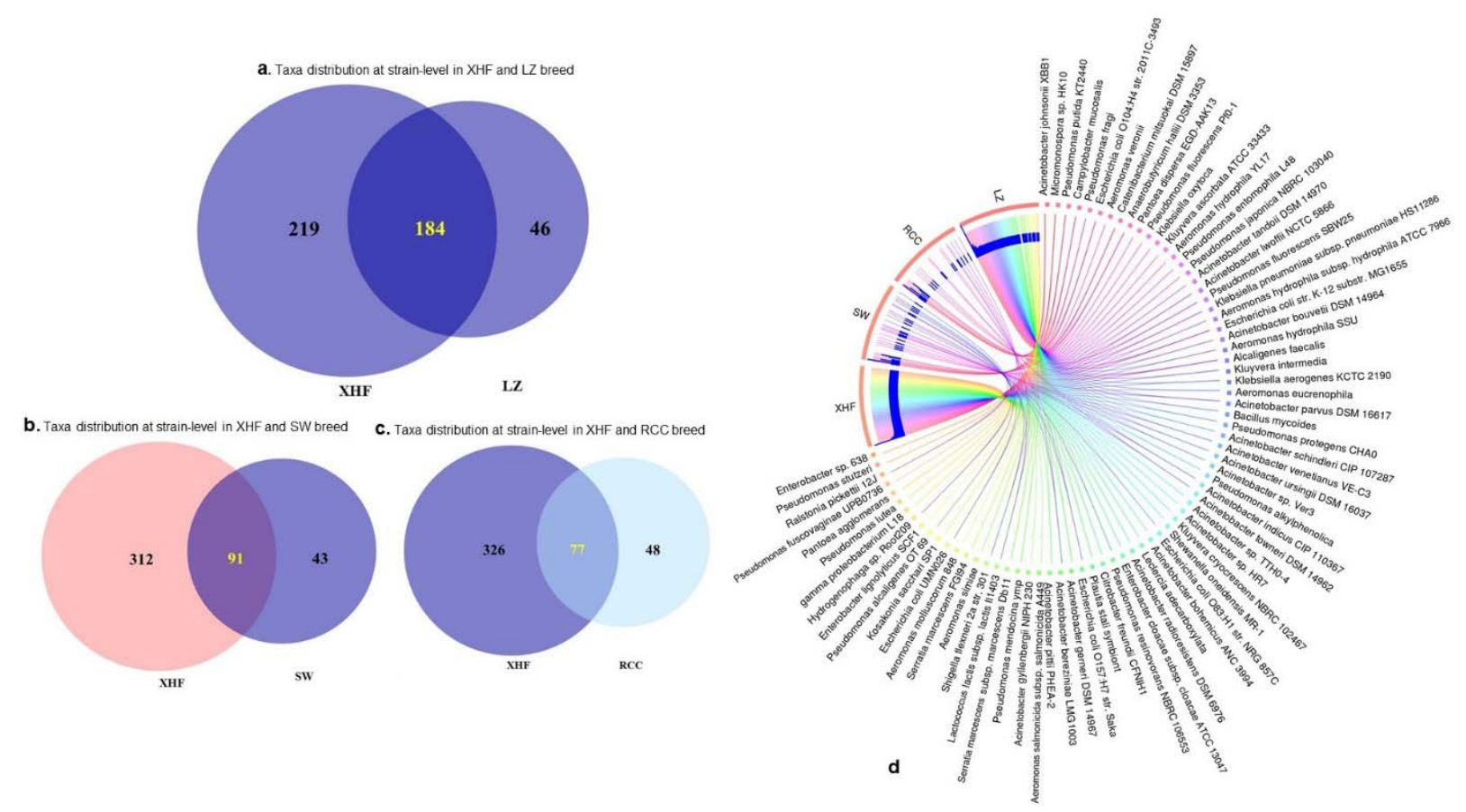

Fig. 3 Strain-level bovine CM microbiome diversity in four different breeds (Local Zebu, LZ; Red Chattogram Cattle, RCC; Sahiwal, SW; Crossbred Holstein Friesian, XHF) of cows through PathoScope (PS) analysis. a) Venn diagrams representing the core unique and shared microbiomes of bovine clinical mastitis (CM) in XHF and LZ breeds while b) and c) Venn diagrams showing the unique and shared bacterial strains in XHF and SW and XHF and RCC breeds, respectively. Microbiome sharing between the conditions are indicated by yellow color. d) The circular plot illustrates the relative abundance of the top $75 \mathrm{CM}$ causing bacterial strains in CM milk samples obtained from XHF, LZ, SW and RCC dairy breeds. Taxa in the respective breed of cows are represented by different colored ribbons, and the inner blue bars indicate their respective relative abundances. The XHF cows had the highest number of microbial strains followed by LZ, SW and RCC. This breed specific association revealed that 45.66, 22.58 and $19.11 \%$ of the detected bacterial strains in CM milk collected from LZ, SW and RCC cows, respectively, were also seen in the CM milk microbiome of XHF cows. The relative abundance bacterial strains in four breeds is also available in Supplementary Data 1. 

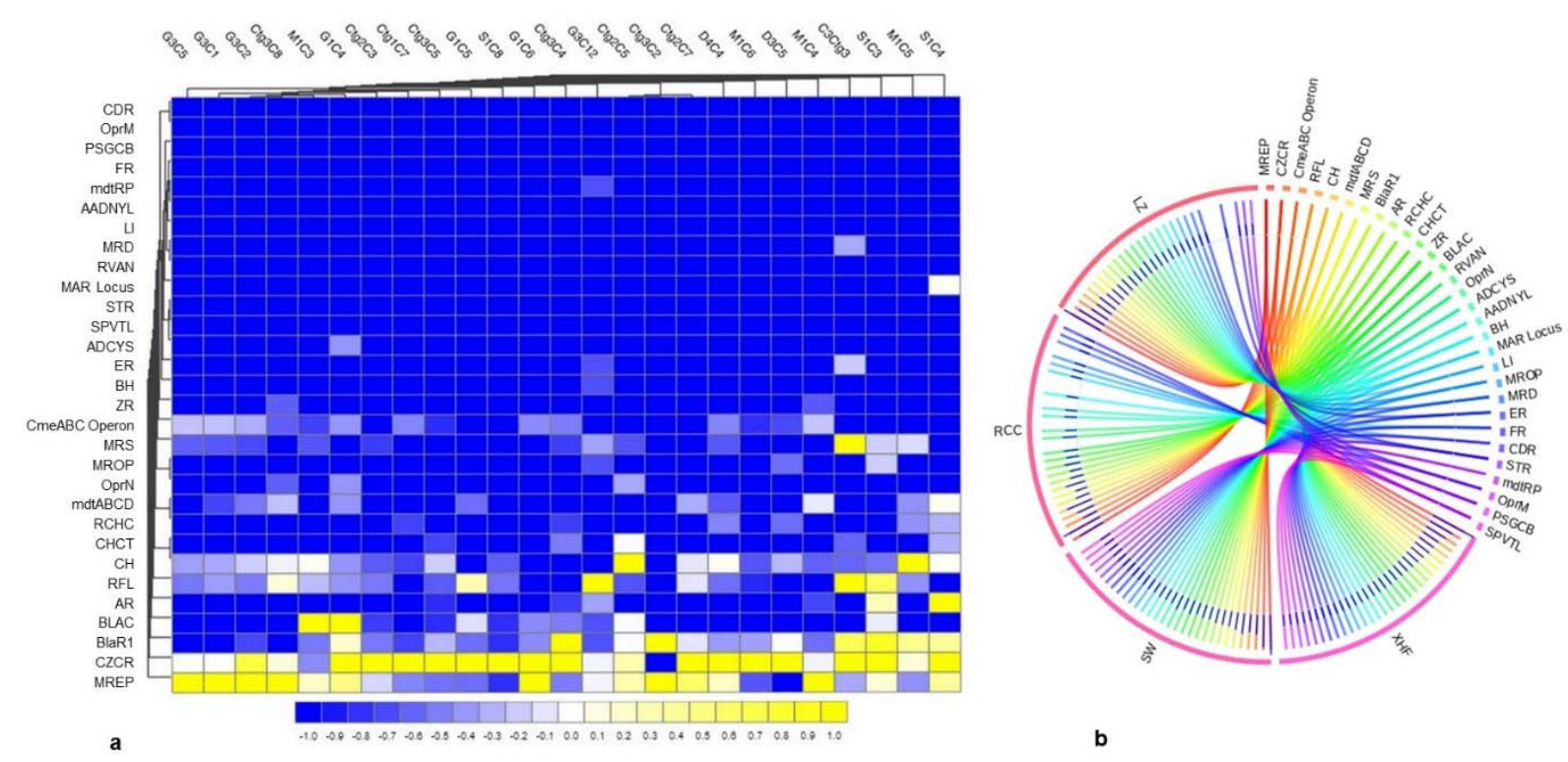

Fig. 4 Projection of the resistance to antibiotic and toxic compounds (RATC) genes in bovine clinical mastitis (CM) pathogens. a) Heatmap showing the hierarchical clustering of 30 different RATC genes detected in CM associated microbiomes of $25 \mathrm{CM}$ milk samples as measured at level-3 of SEED subsystems in MG-RAST pipeline. The relative abundance of these genes significantly correlated (Pearson correlation, $p=0.002$ ) with the relative abundance of the bacterial taxa found in these samples. The color bar at the bottom represents the relative abundance of putative genes and expressed as a value between -1 (low abundance) and 1 (high abundance). The yellow color indicates the more abundant patterns, while blue cells for less abundant RATC gene in that particular sample. b) The circular plot illustrates the diversity and relative abundance of the RATC genes detected among the microbiomes of the four different breeds (Local Zebu, LZ; Red Chattogram Cattle, RCC; Sahiwal, SW; Crossbred Holstein Friesian, XHF) of cows through SEED subsystems analysis. We found no significant correlation between the resistome and microbiome diversity in different breeds $(p=0.692)$. The association of the RATC genes according to breeds is shown by different colored ribbons and the relative 
abundances these genes are represented by inner blue colored bars. Part of the RATC functional groups are shared among microbes of the four breeds (XHF, LZ, SW and RCC), and some are effectively undetected in the microbiomes of the other breeds. Abbreviations: MREP, multidrug resistance efflux pumps; CZCR, cobalt-zinc-cadmium resistance; BlaR, BlaR1 family regulatory sensor-transducer disambiguation; BLAC, beta-lactamase resistance; AR, arsenic resistance; RFL, resistance to fluoroquinolones; $\mathrm{CH}$, copper homeostasis; $\mathrm{CHCT}$, copper homeostasis: copper tolerance; $\mathrm{RCHC}$, resistance to chromium compounds; mdtABCD, the mdtABCD multidrug resistance cluster; OprN, mexe-mexf-oprn multidrug efflux system; MROP, mercury resistance to operon; MRS, methicillin resistance in Staphylococci; CmeABC Operon, multidrug efflux pump in Campylobacter jejuni; ZR, zinc resistance; BH, bile hydrolysis; ER, erythromycin resistance; ADCYS, adaptation to d-cysteine; SPVTL, Streptococcus pneumoniae vancomycin tolerance locus; STR, Streptothricin resistance; MAR Locus, multiple antibiotic resistance to locus; RVAN, resistance to vancomycin; MRD, mercuric reductase; LI, lysozyme inhibitors; AADNYL, aminoglycoside adenylyltransferases; mdtRP, multidrug resistance operon mdtRP of Bacillus; FR, Fosfomycin resistance; PSGCB, polymyxin synthetase gene cluster in Bacillus; OprM, mexA-mexB-oprm multidrug efflux system; CDR, cadmium resistance. Additional information is also available in Supplementary Data 2. 

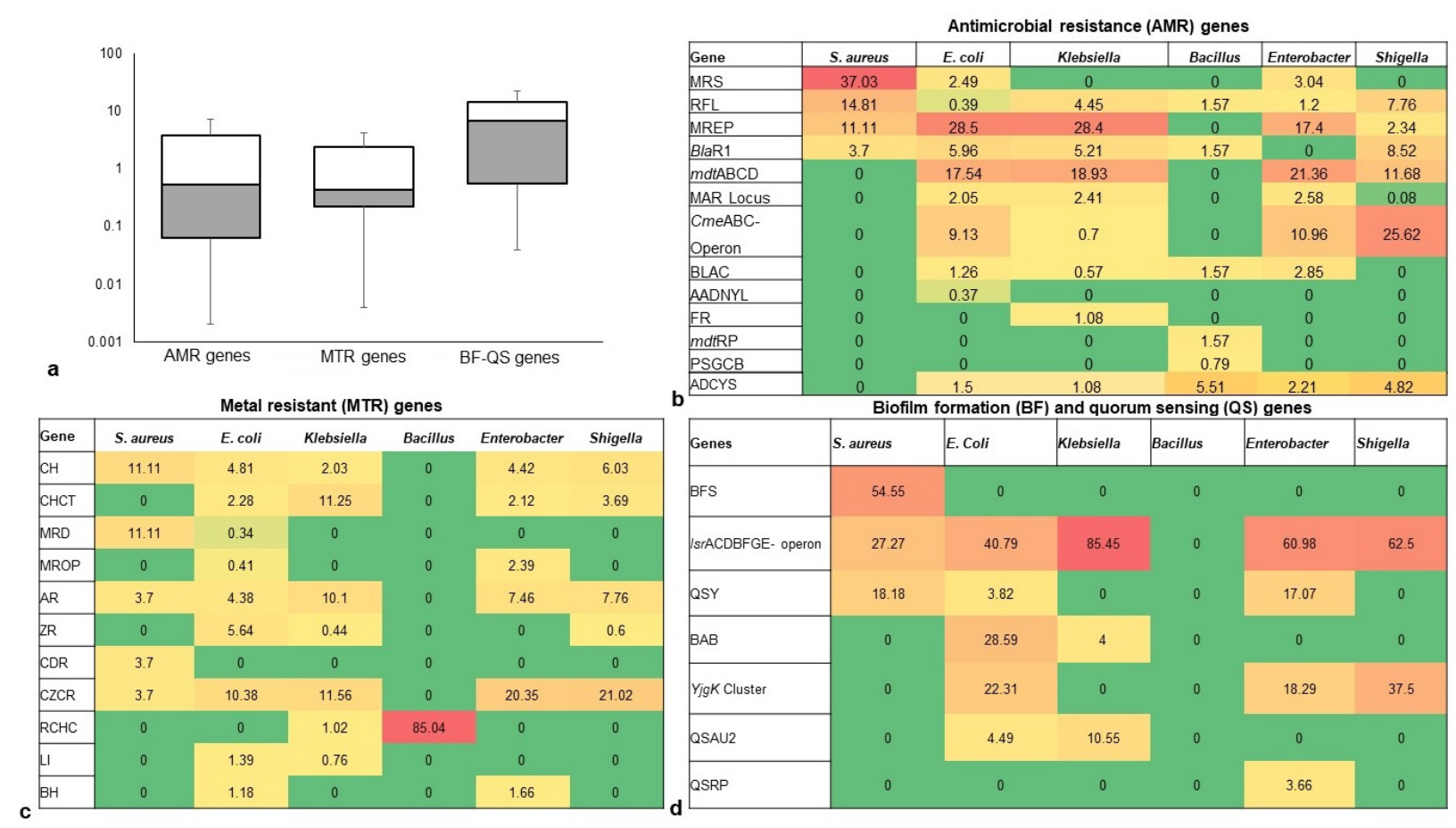

Fig. 5 Heatmap comparison of antibiotics, metals, biofilm formation and quorum sensing genes

found in the metagenome sequences (WMS) of six CM causing bacteria through SEED subsystems analysis in MG-RAST pipeline. a) Diversity and relative abundance of the antimicrobial resistance (AMR), metal resistance (MTR), and biofilm formation (BF) and quorum sensing (QS) genes varied significantly (Kruskal-Wallis test, $p=0.029$ ) among the study bacteria. b) Relative abundance of AMR genes, c) Relative abundance of MTR genes d) Relative abundance of BF-QS genes. Values are colored in shades of green to yellow to red, indicating low (absent), medium and high abundance, respectively. Abbreviations: MRS, methicillin resistance in Staphylococci; RFL, resistance to fluoroquinolones; MREP, multidrug resistance to efflux pumps; BlaR, BlaR1 family regulatory sensor-transducer disambiguation; mdtABCD, the mdtABCD multidrug resistance cluster; MAR Locus, multiple antibiotic resistance; CmeABC Operon, Multidrug efflux pump in Campylobacter jejuni; BLAC, beta-lactamase resistance; AADNYL, aminoglycoside adenylyltransferases (Gentamycin resistance); FR, Fosfomycin resistance; mdtRP, multidrug resistance operon mdtRP of Bacillus; PSGCB, polymyxin 
synthetase gene cluster in Bacillus; BFS, biofilm formation in Staphylococcus, 1srACDBFGE operon, autoinducer 2 (AI-2) transport and processing; QSY, quorum sensing in Yersinia; BAB, biofilm adhesion biosynthesis; $Y j g \mathrm{~K}$ cluster, protein $\mathrm{YjgK}$ cluster linked to biofilm formation; QSAU2, quorum sensing: autoinducer-2 synthesis; QSRP, quorum sensing regulation in Pseudomonas; $\mathrm{CH}$, copper homeostasis; CHCT, copper homeostasis: copper tolerance; MRD, mercuric reductase; MROP, mercury resistance to operon; AR, arsenic resistance; ZR, zinc resistance; CDR, cadmium resistance; CZCR, cobalt-zinc-cadmium resistance; ADCYS, adaptation to d-cysteine; RCHC, resistance to chromium compounds; LI, lysozyme inhibitors; $\mathrm{BH}$, bile hydrolysis. More details about these genes can be found in the text and Supplementary Data 2. 


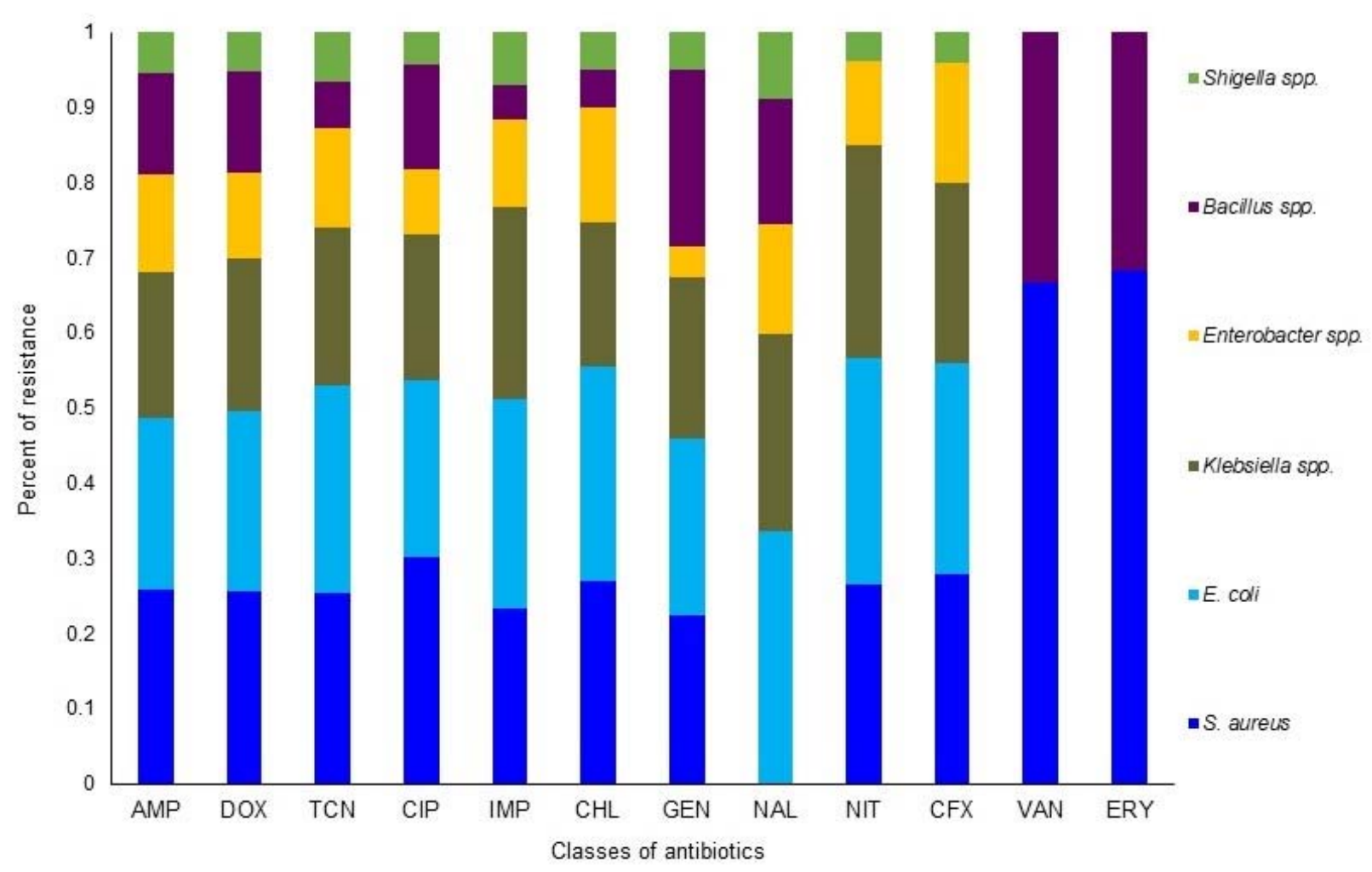

Fig. 6 Antibiotic resistance pattern of bovine clinical mastitis pathogens by disk diffusion method. The antimicrobial resistance (AMR) patterns of the six bacteria obtained from $221 \mathrm{CM}$ isolates (S. aureus, 56; E. coli, 54; Klebsiella spp., 42; Enterobacter spp., 26; Bacillus spp., 31; Shigella spp., 12) for twelve commonly used antibiotics from nine different groups/classes. Abbreviations: AMP, Ampicillin; DOX, Doxycycline; TCN, Tetracycline; CIP, Ciprofloxacin; IMP, Imipenem; CHL, Chloramphenicol; GEN, Gentamycin; NAL, Nalidixic acid; NIT, Nitrofurantoin; CFX, Cefoxitin; VAN, Vancomycin; ERY, Erythromycin. More details about AMR profiles can be found in the text and in Table 1. 

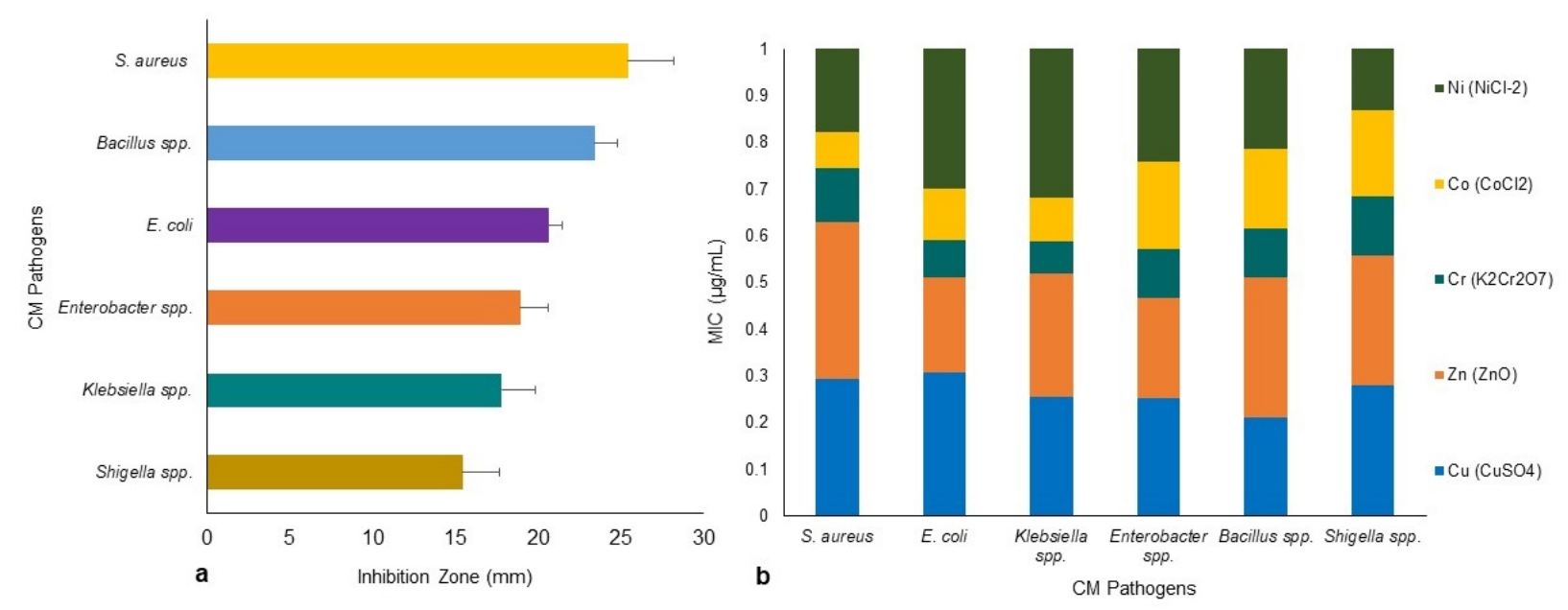

Fig. 7 Antibacterial activity of heavy metals: $\mathrm{Cu}$ (CuSO4), $\mathrm{Zn}(\mathrm{ZnO}), \mathrm{Cr}$ (K2Cr2O7), Co $(\mathrm{CoCl} 2)$ and $\mathrm{Ni}(\mathrm{NiCl})$ against bovine $\mathrm{CM}$ pathogens. a) Zone of inhibition (ZOI, mm) for six CM causing bacteria, each bar representing the mean values (values given horizontal axis of the bars, mm) and standard deviation error bar (SD error bar) for each bacterium. b) Minimal inhibitory concentration (MIC) (expressed as $\mu \mathrm{g} / \mathrm{mL}$ ) of the tested metals against representative genera/species as determined by agar well diffusion and tube dilution methods. 


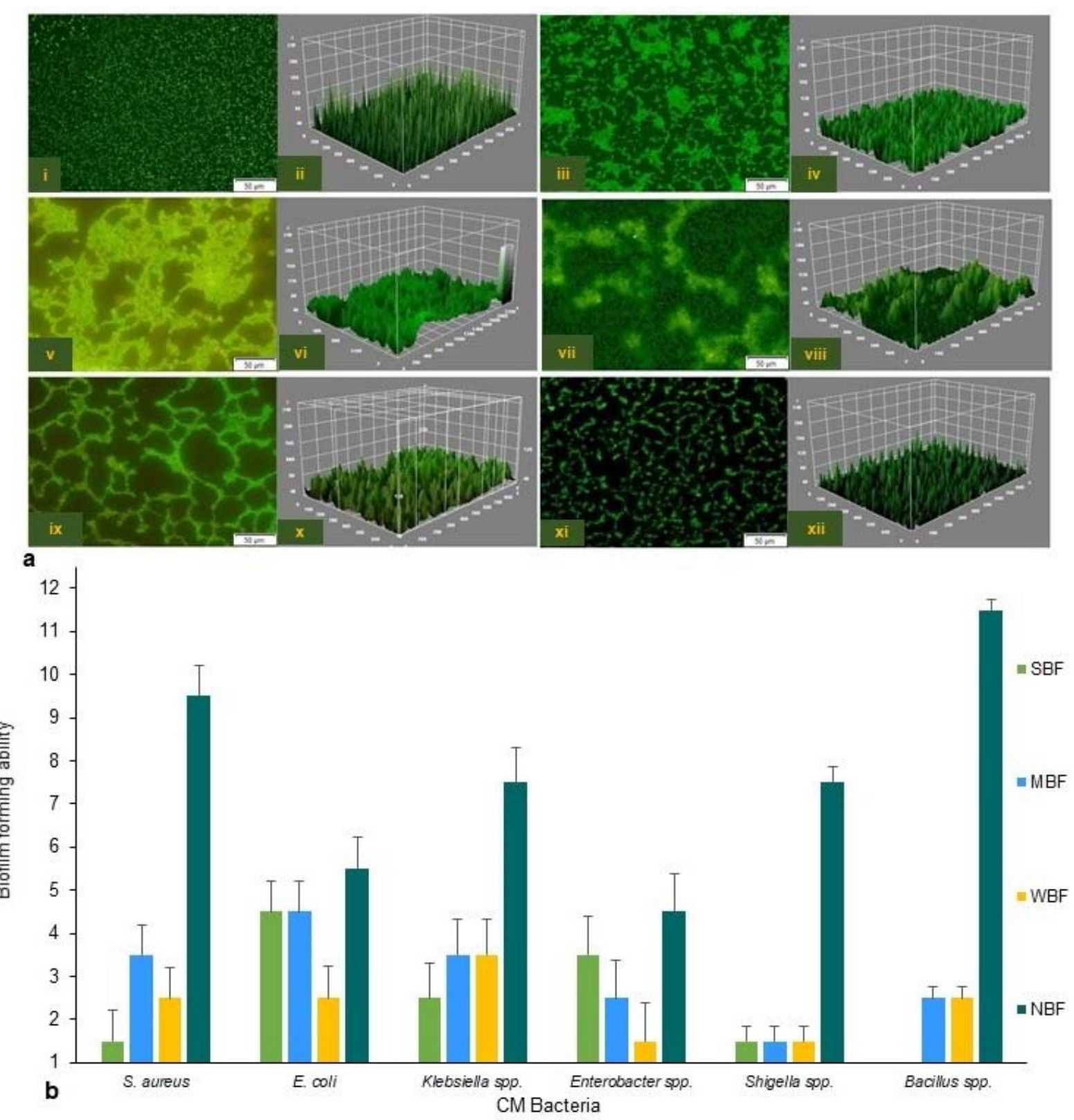

Fig. 8 Biofilm formation (BF) ability of the six $\mathrm{CM}$ causing pathogens. BF assays was performed with solubilized crystal violet (CV) in a plate reader at $600 \mathrm{~nm}$ using $30 \%$ acetic acid in water as the blank and TSB as negative control. a) Confocal fluorescence images (2D and 3D) of S. aureus (i,ii), E. coli (iii,iv), Klebsiella spp. (v,vi), Enterobacter spp. (vii,viii), Bacillus spp. (ix,x) and Shigella spp. (xi,xii). Scale bars are indicated in $\mu \mathrm{m}$. b) Category of the biofilm formation by six CM causing bacteria. The BF ability of the tested bacteria were classified as 
follows: NBF, non-biofilm formers optical density $(\mathrm{OD}) \leq$ optical density cut-off (ODc); WBF, weak biofilm formers (ODc < OD $\leq 2 \times$ ODc); MBF, moderate biofilm formers $(2 \times$ ODc $<$ OD $\leq 4 \times$ ODc), SBF, strong biofilm formers (OD > 4 x ODc). The ODc value was set as 0.045 and the mean OD of the negative control was $0.039 \pm 0.002$. Thus, bacterial biofilms were divided into breakpoint categories; OD $<0.045$ non-biofilm producers; OD $\geq 0.046$ but $\leq 0.090$ weak biofilm producers; $\geq$ OD $0.091-\leq 0.180$ moderate or partial biofilm producers; >0.181 strong biofilm producers. The results are presented as the mean $\pm \mathrm{SD}$, and post hoc Bonferroni test was used to compare the biofilm OD600 mean values $(p<0.05)$. 


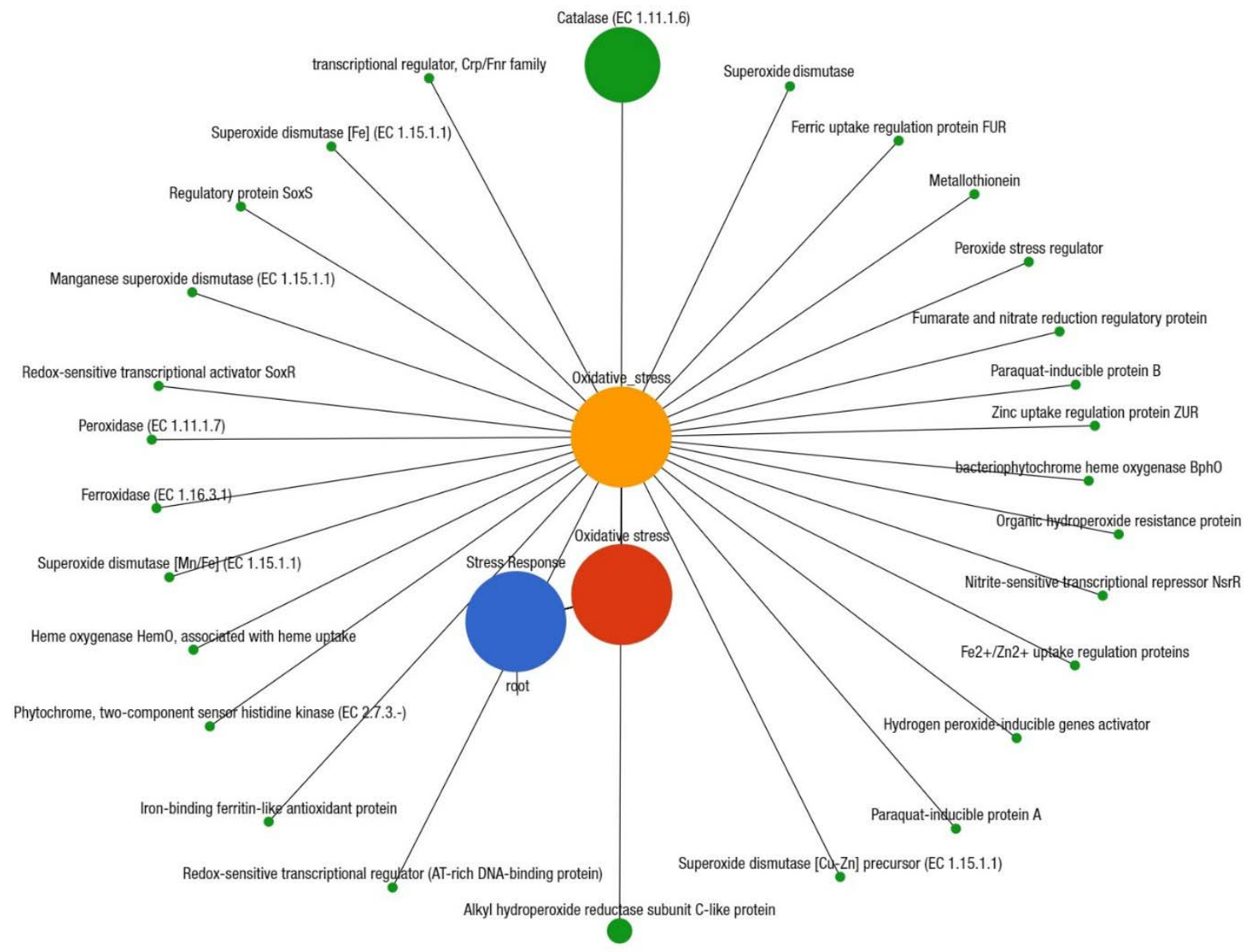

Fig. 9 Projection of the clinical mastitis (CM) milk metagenome onto KEGG pathways. The whole metagenome sequencing (WMS) reveals significant differences (Kruskal-Wallis test, $p=0.001$ ) in functional microbial pathways. A total of 28 genes associated with oxidative stress were found in $\mathrm{CM}$ microbiomes. Black lines with green circles delineate the distribution of the stress related genes according to their class across the CM metagenome. The diameter of the circles indicates the relative abundance of the respective genes. 


\section{Resistome diversity in bovine clinical mastitis microbiome, a signature concurrence}

M. Nazmul Hoque, Arif Istiaq, Rebecca A. Clement, Keylie M. Gibson, Otun Saha, Ovinu Kibria Islam, Ruhshan Ahmed Abir,

Munawar Sultana, AMAM Zonaed Siddiki, Keith A. Crandall, M. Anwar Hossain

Table 1: Antibiotic resistance pattern of bacteria [n (\%) of isolates] associated with bovine clinical mastitis (CM).

\begin{tabular}{lcccccccc}
\hline Antibiotic & $\begin{array}{l}\text { Content } \\
\text { per disk }\end{array}$ & $\begin{array}{c}\text { Breakpoint to } \\
\text { declare resistance }(\leq)\end{array}$ & $\begin{array}{c}\text { S. aureus } \\
(\mathrm{n}=56)\end{array}$ & $\begin{array}{c}\text { E. coli } \\
(\mathrm{n}=54)\end{array}$ & $\begin{array}{c}\text { Klebsiella } \\
\text { spp. } \\
(\mathrm{n}=42)\end{array}$ & $\begin{array}{c}\text { Enterobacter } \\
\text { spp. }(\mathrm{n}=26)\end{array}$ & $\begin{array}{c}\text { Bacillus } \\
\text { spp. } \\
(\mathrm{n}=31)\end{array}$ & $\begin{array}{c}\text { Shigella } \\
\text { spp. (n=12) }\end{array}$ \\
\hline AMP & $10 \mu \mathrm{g}$ & $28 \mathrm{~mm}$ & $48(85.71)$ & $42(77.78)$ & $36(85.71)$ & $24(92.30)$ & $25(80.64)$ & $10(83.33)$ \\
DOX & $30 \mu \mathrm{g}$ & $23 \mathrm{~mm}$ & $49(87.50)$ & $46(85.18)$ & $39(92.86)$ & $22(84.61)$ & $26(83.87)$ & $10(83.33)$ \\
TCN & $30 \mu \mathrm{g}$ & $23 \mathrm{~mm}$ & $46(82.14)$ & $50(92.59)$ & $38(90.48)$ & $24(92.30)$ & $11(35.48)$ & $12(100)$ \\
CIP & $10 \mu \mathrm{g}$ & $20 \mathrm{~mm}$ & $28(50.0)$ & $22(40.74)$ & $18(42.86)$ & $8(30.77)$ & $13(41.94)$ & $4(33.33)$ \\
IMP & $10 \mu \mathrm{g}$ & $22 \mathrm{~mm}$ & $10(17.86)$ & $12(22.22)$ & $11(26.19)$ & $5(19.23)$ & $2(6.45)$ & $3(25.0)$ \\
CHL & $30 \mu \mathrm{g}$ & $12 \mathrm{~mm}$ & $32(57.14)$ & $34(62.96)$ & $23(54.76)$ & $18(69.23)$ & $6(19.35)$ & $6(50.00)$ \\
GEN & $10 \mu \mathrm{g}$ & $12 \mathrm{~mm}$ & $22(39.28)$ & $23(42.60)$ & $21(50.0)$ & $4(15.38)$ & $23(74.19)$ & $5(41.67)$ \\
NAL & $30 \mu \mathrm{g}$ & $16 \mathrm{~mm}$ & ND & $46(85.18)$ & $36(85.71)$ & $20(76.92)$ & $23(74.19)$ & $12(100)$ \\
NIT & $10 \mu \mathrm{g}$ & $64 \mathrm{~mm}$ & $28(50.0)$ & $32(59.25)$ & $30(71.42)$ & $12(46.15)$ & ND & $4(33.33)$ \\
CFX & $30 \mu \mathrm{g}$ & $24 \mathrm{~mm}$ & $14(25.0)$ & $14(25.0)$ & $12(28.57)$ & $8(30.77)$ & ND & $2(16.67)$ \\
VAN & $30 \mu \mathrm{g}$ & $20 \mathrm{~mm}$ & $12(21.42)$ & ND & ND & ND & $6(19.35)$ & ND \\
ERY & $15 \mu \mathrm{g}$ & $20 \mathrm{~mm}$ & $41(73.21)$ & ND & ND & ND & $19(61.29)$ & ND
\end{tabular}

n: total number of isolates tested; ND: Not done; AMP: Ampicillin; DOX: Doxycycline; TCN: Tetracycline; CIP: Ciprofloxacin; IMP Imipenem; CHL: Chloramphenicol; GEN: Gentamycin; NAL: Nalidixic acid; NIT: Nitrofurantoin; CFX: Cefoxitin; VAN: Vancomycin; ERY: Erythromycin. 